\title{
Holographic phase transition from dyons in an AdS black hole background
}

\author{
A. R. Lugo ${ }^{a *}$ E. F. Moreno ${ }^{b}$ and F. A. Schaposnik ${ }^{a \dagger}$ \\ ${ }^{a}$ Departamento de Física-IFLP, Universidad Nacional de La Plata \\ C.C. 67, 1900 La Plata, Argentina \\ ${ }^{b}$ Department of Physics, West Virginia University \\ Morgantown, West Virginia 26506-6315, U.S.A.
}

October 29, 2018

\begin{abstract}
We construct a dyon solution for a Yang-Mills-Higgs theory in a 4 dimensional Schwarzschild-anti-de Sitter black hole background with temperature T. We then apply the AdS/CFT correspondence to describe the strong coupling regime of a $2+1$ quantum field theory which undergoes a phase transition exhibiting the condensation of a composite charge operator below a critical temperature $T_{c}$.
\end{abstract}

\section{Introduction}

It has recently been observed that the AdS/CFT correspondence [1]-[3] provides a powerful tool to study quantum critical dynamics in condensed matter models for superconductivity, superfluidity and Hall effect [4]-[10]. In this way, using the dual classical gravity description, correlation functions in strongly interacting systems can be calculated and the relevant physical

${ }^{*}$ Associated with CONICET

${ }^{\dagger}$ Associated with CICBA 
properties can be determined in a relatively simple way, which has motivated an intense activity in the field (some works related to the present approach are listed in [1] ).

Temperature is introduced in the gravity dual through the presence of a Schwarzschild-AdS black hole, either added as a background or resulting from back reaction. Apart from gauge fields, the models referred above include minimally coupled scalar matter fields. In the Abelian case the ansatz for gauge fields included an electrostatic potential $A_{0}$ which is spatially independent at the boundary as also is the case for the scalar. Spatially dependent droplet and vortex solutions have been also studied in [8]-[9] and in the nonAbelian case non-trivial gauge-field components were considered [7],[10]. In general, such classical solutions in the bulk exist below a certain critical temperature leading to thermodynamically favored phases in the the strongly coupled quantum field theory on the boundary.

Following the ideas described above, we consider in the present paper a model not yet studied: a dyon solution for Yang-Mills-Higgs theory in a 4 dimensional Schwarzschild-anti-de Sitter black-hole background metric. We shall be guided by our previous work on monopole and dyon solutions in $\mathrm{AdS}_{4}$ space [12]-[13] where, due to the absence of singularities, the spherically symmetric solutions were constructed in the whole radial domain $\mathbb{R}^{+}$, while in the present case the radial variable extend from the black hole horizon to infinity. The black hole temperature, determined by the horizon, will become the temperature of the field theory defined on the boundary. The matter field will lead to the non-Abelian gauge symmetry breaking condensate while the field strength associated to the surviving $U(1)$ symmetry will correspond to that of a dyon with electric and magnetic charges.

The paper is organized as follows. In section 2 we present the gravityYang-Mills-Higgs model and specialize to the case in which the gravitational equations decouple from matter leading to an AdS-Schwarzschild black hole background for the gauge-matter system. Then in section 3 we analyze the properties of the dyon solution considering both the cases of an $S^{2} \times S^{1}$ and $\mathbb{R}^{2} \times S^{1}$ boundaries. In section 4 we proceed to describe the holography calculation of relevant quantities in the dual field theory defined on the boundary (taken as $\mathbb{R}^{2} \times S^{1}$ ) and present our numerical results. We end with a summary and discussion in section 5 . 


\section{The gravity-Yang-Mills-Higgs system}

\subsection{The model}

We consider a gravity-Yang-Mills-Higgs system with gauge group $S U(2)$ and the scalar field in the adjoint representation, in a 4 dimensional space-time with Minkowski signature $(-,+,+,+)$. The action takes the form

$$
S=S_{G}+S_{Y M}+S_{H}=\int d^{4} x \sqrt{|g|}\left(L_{G}+L_{Y M}+L_{H}\right)
$$

with

$$
\begin{gathered}
L_{G}=\frac{1}{2 \kappa^{2}}(R-2 \Lambda) \\
L_{Y M}=-\frac{1}{4 e^{2}} F_{\mu \nu}^{a} F^{a \mu \nu} \\
L_{H}=-\frac{1}{2} D_{\mu} H^{a} D^{\mu} H^{a}-V(H) \\
V(H)=\frac{\lambda}{4}\left(H^{a} H^{a}-H_{0}^{2}\right)^{2} \\
\kappa^{2} \equiv 8 \pi G_{N}
\end{gathered}
$$

Here $G_{N}$ is the Newton constant, $e$ the gauge coupling and $\Lambda$ the cosmological constant (with our conventions $\Lambda<0$ corresponds, in the absence of matter, to anti-de Sitter space). The field strength $F_{\mu \nu}^{a}(a=1,2,3)$ is defined as

$$
F_{\mu \nu}^{a}=\partial_{\mu} A_{\nu}^{a}-\partial_{\nu} A_{\mu}^{a}+\varepsilon^{a b c} A_{\mu}^{b} A_{\nu}^{c}
$$

and the covariant derivative $D_{\mu}$ acting on the Higgs triplet $H^{a}$ is given by

$$
D_{\mu} H^{a}=\partial_{\mu} H^{a}+\varepsilon^{a b c} A_{\mu}^{b} H^{c}
$$

The equations of motion that follow from (11) are

$$
\begin{aligned}
E_{\mu \nu}+\Lambda g_{\mu \nu} & =\kappa^{2}\left(T_{\mu \nu}^{Y M}+T_{\mu \nu}^{H}\right) \\
D_{\rho} D^{\rho} H^{a} & =\frac{\delta V(H)}{\delta H^{a}} \\
\frac{1}{e^{2}} D^{\rho} F_{\mu \rho}^{a} & =\varepsilon^{a b c}\left(D_{\mu} H^{b}\right) H^{c}
\end{aligned}
$$


where $E_{\mu \nu}$ is the Einstein tensor and the matter energy-momentum tensor $T_{\mu \nu}$,

$$
T_{\mu \nu}=-2 \frac{\delta S}{\delta g^{\mu \nu}}
$$

is given by

$$
\begin{aligned}
T_{\mu \nu}^{Y M} & =\frac{1}{e^{2}} F_{\mu \rho}^{a} F_{\nu}^{a \rho}+g_{\mu \nu} L_{Y M} \\
T_{\mu \nu}^{H} & =D_{\mu} H^{a} D_{\nu} H^{a}+g_{\mu \nu} L_{H}
\end{aligned}
$$

The most general static spherically symmetric form for the metric in 3 spatial dimensions together with the t'Hooft-Polyakov-Julia-Zee ansatz for the gauge and Higgs fields in the usual vector notation reads [12]-[13]

$$
\begin{aligned}
g & =-\mu(x) A(x)^{2} d^{2} t+\mu(x)^{-1} d^{2} r+r^{2} d^{2} \Omega_{2} \\
\vec{A} & =d t e H_{0} J(x) \check{e}_{r}-d \theta(1-K(x)) \check{e}_{\varphi}+d \varphi(1-K(x)) \sin \theta \check{e}_{\theta} \\
\vec{H} & =H_{0} H(x) \check{e}_{r}
\end{aligned}
$$

where we have introduced the dimensionless radial coordinate $x \equiv e H_{0} r$ and we denote the standard spherical unit vectors as $\check{e}_{r}, \check{e}_{\varphi}, \check{e}_{\theta}$. $H_{0}$ sets the mass scale $\left(\left[H_{0}\right]=m^{1}\right)$.

Using this ansatz, the equations of motion take the form

$$
\begin{aligned}
& (x \mu(x))^{\prime}=1+3 \gamma_{0}^{2} x^{2}-\kappa^{2} H_{0}^{2}\left(\mu(x) V_{1}+V_{2}+\frac{x^{2}}{2} \frac{J^{\prime}(x)^{2}}{A(x)^{2}}\right. \\
& \left.+\frac{J(x)^{2} K(x)^{2}}{\mu(x) A(x)^{2}}\right) \\
& x A^{\prime}(x)=\kappa^{2} H_{0}^{2}\left(V_{1}+\frac{J(x)^{2} K(x)^{2}}{\mu(x)^{2} A(x)^{2}}\right) A(x) \\
& \left(\mu(x) A(x) K^{\prime}(x)\right)^{\prime}=A(x) K(x)\left(\frac{K(x)^{2}-1}{x^{2}}+H(x)^{2}\right. \\
& \left.-\frac{J(x)^{2}}{\mu(x) A(x)^{2}}\right) \\
& \mu(x) A(x)\left(\frac{x^{2} J^{\prime}(x)}{A(x)}\right)^{\prime}=2 J(x) K(x)^{2} \\
& \left(x^{2} \mu(x) A(x) H^{\prime}(x)\right)^{\prime}=A(x) H(x)\left(2 K(x)^{2}+\frac{\lambda}{e^{2}} x^{2}\left(H(x)^{2}-1\right)\right)
\end{aligned}
$$


where, for convenience, we have defined the dimensionless parameter

$$
\gamma_{0}^{2} \equiv-\frac{\Lambda}{3 e^{2} H_{0}^{2}}=\frac{1}{L^{2} e^{2} H_{0}^{2}} \quad, \quad L^{2} \equiv-\frac{3}{\Lambda}
$$

and

$$
\begin{aligned}
& V_{1}=K^{\prime}(x)^{2}+\frac{x^{2}}{2} H^{\prime}(x)^{2} \\
& V_{2}=\frac{\left(K(x)^{2}-1\right)^{2}}{2 x^{2}}+\frac{\lambda}{4 e^{2}} x^{2}\left(H(x)^{2}-1\right)^{2}
\end{aligned}
$$

\subsection{The AdS-Schwarzschild black hole background}

In the $\kappa^{2} \rightarrow 0$ limit the gravitational equations in system (13) decouple from the matter ones leading to a solution of the metric $g$ of the form

$$
g=-\mu_{0}(x) d^{2} t+\mu_{0}^{-1}(x) d^{2} r+r^{2} d^{2} \Omega_{2}
$$

with

$$
\mu_{0}(x)=1+\gamma_{0}^{2} x^{2}-\frac{R^{3}}{\gamma_{0} x}
$$

Here $R$ is a dimensionless parameter related to the black hole mass. For $R \neq 0$ the metric defined in (16) has an horizon at $x=x_{h}$, i.e.,

$$
\mu_{0}\left(x_{h}\right)=0
$$

with $\mu_{0}^{\prime}\left(x_{h}\right) \neq 0$. Written in terms of the parameters of the model $x_{h}$ reads

$$
\gamma_{0} x_{h}=\left(\sqrt{\frac{R^{6}}{4}+\frac{1}{27}}+\frac{R^{3}}{2}\right)^{1 / 3}-\left(\sqrt{\frac{R^{6}}{4}+\frac{1}{27}}-\frac{R^{3}}{2}\right)^{1 / 3}
$$

In order to compute the black hole temperature one uses the standard recipe [16] leading to no conical singularity at the horizon after Wick rotation to the Euclidean time $\tau_{E} \equiv i t$. Hence, given (17) and imposing

$$
\tau_{E} \sim \tau_{E}+\beta
$$

one identifies the black hole temperature as

$$
T \equiv \frac{1}{\beta}=\frac{\left|\mu_{0}^{\prime}\left(x_{h}\right)\right| e H_{0}}{4 \pi}=\frac{1}{4 \pi L}\left(3 \gamma_{0} x_{h}+\frac{1}{\gamma_{0} x_{h}}\right)
$$




\section{The dyon in the black hole background}

\subsection{Properties of the solution}

From here on we shall consider, for simplicity, the BPS limit of the potential (5) which correspond to $\lambda / e^{2}=0$ with $H_{0}$ fixed. Taking the black hole metric (17) as a background we are left with the system

$$
\begin{aligned}
& \left(\mu_{0}(x) K^{\prime}(x)\right)^{\prime}=K(x)\left(\frac{K(x)^{2}-1}{x^{2}}+H(x)^{2}-\frac{J(x)^{2}}{\mu_{0}(x)}\right) \\
& \mu_{0}(x)\left(x^{2} J^{\prime}(x)\right)^{\prime}=2 J(x) K(x)^{2} \\
& \left(x^{2} \mu_{0}(x) H^{\prime}(x)\right)^{\prime}=2 H(x) K(x)^{2}
\end{aligned}
$$

We shall look for a solution to (22)-(24) regular at the horizon

$$
K(x), H(x), J(x) /\left(x-x_{h}\right) \quad \text { regular at } x_{h}
$$

so that

$$
\begin{aligned}
H(x) & =h_{0}+h_{1}\left(x-x_{h}\right)+\mathcal{O}\left[\left(x-x_{h}\right)^{2}\right] \\
K(x) & =k_{0}+k_{1}\left(x-x_{h}\right)+\mathcal{O}\left[\left(x-x_{h}\right)^{2}\right] \\
J(x) & =j_{h}\left(x-x_{h}\right)+\mathcal{O}\left[\left(x-x_{h}\right)^{2}\right]
\end{aligned}
$$

Concerning $x \rightarrow \infty$, the asymptotic behavior takes the form

$$
\begin{array}{rlr}
|\vec{H}(x)| & =H_{0}\left(1-\frac{H_{1}}{x^{3}}+\frac{H_{2}}{x^{5}}+\frac{H_{3}}{x^{4+2 \nu}}+\cdots\right) \\
K(x) & =\frac{K_{1}}{x^{\nu+1}}+\frac{K_{3}}{x^{\nu+3}},+\cdots \quad \nu \in \mathbb{R} \quad \text { as } \mathrm{x} \rightarrow \infty \\
J(x) & =J_{0}+\frac{J_{1}}{x}+\ldots &
\end{array}
$$

Such behavior is consistent with the equation of motion (22) provided that the following condition holds

$$
\frac{1}{\gamma_{0}^{2}}=\nu(\nu+1)
$$

Equation (30) has two solutions,

$$
\nu_{ \pm}=-\frac{1}{2} \pm \frac{1}{2} \sqrt{1+\frac{4}{\gamma_{0}^{2}}}
$$


Only the $\nu_{+}$root gives an acceptable asymptotic behavior for $K(x)$ so that

$$
K(x) \sim \frac{K_{1}}{x^{\nu+1}}=\frac{K_{1}}{\left.x^{\frac{1}{2}\left(1+\sqrt{1+\frac{4}{\gamma_{0}^{2}}}\right.}\right)} \quad \text { as } x \rightarrow \infty
$$

Being the $S U(2)$ gauge symmetry of the Lagrangian spontaneously broken by the expectation value of the scalar field, we shall define the field strength associated with the surviving $U(1)$ symmetry as in [15]

$$
\mathcal{F}_{\mu \nu}^{U(1)} \equiv \frac{H^{a}}{H_{0}} F_{\mu \nu}^{a}
$$

From this expression one finds the U(1) magnetic and electric fields

$$
B^{i}=\frac{1}{2} \frac{\varepsilon^{i j k}}{\sqrt{g^{(3)}}} \mathcal{F}_{j k}^{U(1)}, \quad E_{i}=\mathcal{F}_{i 0}^{U(1)}
$$

and the magnetic and electric charges

$$
\begin{aligned}
Q_{m} & =\int_{V} d^{3} x \sqrt{g^{(3)}} \nabla_{i}^{(3)} B^{i} \\
Q_{e} & =\int_{V} d^{3} x \sqrt{g^{(3)}} \nabla_{i}^{(3)} E^{i}
\end{aligned}
$$

where $\nabla_{i}^{(3)}$ is the 3 -dimensional covariant derivative. In order to compute $Q_{m}$ one just needs the asymptotic value of $\mathcal{F}_{i j}^{U(1)}$ since eqs.(34)-(35) imply

$$
Q_{m}=\frac{1}{2} \int_{\partial V=S^{2}} \varepsilon^{i j k} \mathcal{F}_{j k}^{U(1)} d S_{i}
$$

Now, from the asymptotic conditions (29) one has

$$
\varepsilon^{i j k} \mathcal{F}_{j k}^{U(1)} \sim \frac{1}{x^{2}} \check{e}_{r} \delta^{i r} \quad \text { for } x \rightarrow \infty
$$

and then

$$
Q_{m}=-\frac{1}{e} \int_{S^{2}} d \Omega=-\frac{4 \pi}{e}
$$

As expected, the spherically symmetric solution has a quantized $n=-1$ magnetic charge (in units of $4 \pi / e$ ). 
Concerning the electric charge, one can see from the asymptotic behavior of $J$, eq. (29), that $J_{0}$ sets the charge scale and $J_{1}$ determines its value. One can write

$$
Q_{e}=\frac{1}{e} \mathcal{Q}\left(e^{2} / \lambda, J_{0}\right)=-\frac{4 \pi}{e} J_{1}
$$

with $\mathcal{Q}$ a dimensionless function to be determined numerically. As for the Julia-Zee flat space dyon, the electric charge is not quantized at the classical level. As we shall see, $J_{0}$ and $J_{1}$ will be identified, through holography, with the chemical potential and charge density of the strongly coupled system respectively.

Let us now find the expression for the dyon action which will be connected with the free energy of the field theory defined on the boundary. Inserting ansatz (12) in eqs.(33)-(4) one obtains for the Yang-Mills and Higgs Lagrangians

$$
\begin{aligned}
L_{Y M} & =-\frac{e^{2} H_{0}^{4}}{2}\left(-\frac{J^{\prime 2}}{A^{2}}-2 \frac{J^{2} K^{2}}{x^{2} \mu A^{2}}+2 \frac{\mu K^{\prime 2}}{x^{2}}+\frac{\left(K^{2}-1\right)^{2}}{x^{4}}\right) \\
L_{H} & =-\frac{e^{2} H_{0}^{4}}{2}\left(\mu H^{\prime 2}+2 \frac{K^{2} H^{2}}{x^{2}}\right)
\end{aligned}
$$

Then, by analytic continuation, the Euclidean action takes the form

$$
S_{E}=-\int_{0}^{\beta} d \tau_{E} \int_{V} d^{3} x \sqrt{g}\left(L_{Y M}+L_{H}\right)
$$

\subsection{From $\mathrm{S}^{2}$ to $\mathbb{R}^{2}$}

As stated in the introduction, our aim is to calculate the thermodynamics of the strongly interacting field theory defined on the boundary $\partial M$ using the dual classical description of the gravity system governed by the action (11) in the 4 dimensional manifold $M$; now, according to (16), $\partial M=S^{2} \times$ $S^{1}$. From the condensed matter viewpoint, it is more relevant to study the finite temperature field theory defined on the plane $\mathbb{R}^{2}$ and not on the sphere $S^{2}$. To this end, one can follow the approach in [17] and proceed to a change coordinates $(t, x, \theta, \varphi) \rightarrow\left(\tau, y, x^{1}, x^{2}\right)$ or, equivalently, $(t, x, \theta, \varphi) \rightarrow$ $(\tau, y, \rho, \varphi)$,

$$
\begin{aligned}
\tau & =\underset{R}{R} t \\
y & =\frac{\gamma_{0}}{R} x
\end{aligned}
$$




$$
\begin{aligned}
& x^{1}=2 R \tan \frac{\theta}{2} \cos \varphi=\rho \cos \varphi \\
& x^{2}=2 R \tan \frac{\theta}{2} \sin \varphi=\rho \sin \varphi
\end{aligned}
$$

together with the field redefinitions

$$
\begin{aligned}
\tilde{A}(y) & =A(x) \\
\tilde{\xi}(y) & =R^{-2} \xi(x) \\
\tilde{H}(y) & =H(x) \\
\tilde{K}(y) & =R^{-1} K(x) \\
\tilde{J}(y) & =R^{-1} J(x)
\end{aligned}
$$

where $R$ was introduced in (17). After this change, ansatz (12) becomes

$$
\begin{aligned}
g & =-\tilde{f}_{R}(y) \tilde{A}(y)^{2} d^{2} \tau+L^{2} \frac{d^{2} y}{\tilde{f}_{R}(y)}+L^{2} y^{2} \frac{d^{2} \vec{x}}{\left(1+\frac{\rho^{2}}{4 R^{2}}\right)^{2}} \\
\vec{A} & =d \tau \text { e } H_{0} \tilde{J}(y)\left(\frac{4 R \rho}{\rho^{2}+4 R^{2}} \check{e}_{\rho}+\frac{4 R^{2}-\rho^{2}}{\rho^{2}+4 R^{2}} \check{e}_{3}\right)^{2} \\
& +\frac{4 R^{2}}{\rho^{2}+4 R^{2}}\left(\frac{1}{R}-\tilde{K}(y)\right)\left[\left(\frac{4 R^{2}-\rho^{2}}{\rho^{2}+4 R^{2}} \check{e}_{\rho}-\frac{4 R \rho}{\rho^{2}+4 R^{2}} \check{e}_{3}\right) \rho d \varphi\right. \\
& \left.-\check{e}_{\varphi} d \rho\right] \\
\vec{H} & =H_{0} \tilde{H}(y)\left(\frac{4 R \rho}{\rho^{2}+4 R^{2}} \check{e}_{\rho}+\frac{4 R^{2}-\rho^{2}}{\rho^{2}+4 R^{2}} \check{e}_{3}\right)
\end{aligned}
$$

where

$$
\tilde{f}_{R}(y)=f_{R}(y)+\tilde{\xi}(y) \quad, \quad f_{R}(y) \equiv \frac{1}{R^{2}}+y^{2}-\frac{1}{y}
$$

and the equations of motion for the Einstein-Yang Mills-Higgs system, eqs. (13) take the form,

$$
\begin{aligned}
& (y \tilde{\xi}(y))^{\prime}=-\kappa^{2} H_{0}{ }^{2}\left(\tilde{f}_{R}(y) \tilde{V}_{1}+\tilde{V}_{2}+\frac{y^{2}}{2} \frac{\tilde{J}^{\prime}(y)^{2}}{\tilde{A}(y)^{2}}+\frac{\tilde{J}(y)^{2} \tilde{K}(y)^{2}}{\tilde{f}_{R}(y) \tilde{A}(y)^{2}}\right) \\
& y \tilde{A}^{\prime}(y)=\kappa^{2} H_{0}{ }^{2}\left(\tilde{V}_{1}+\frac{\tilde{J}(y)^{2} \tilde{K}(y)^{2}}{\tilde{f}_{R}(y)^{2} \tilde{A}(y)^{2}}\right) \tilde{A}(y) \\
& \left(\tilde{f}_{R}(y) \tilde{A}(y) \tilde{K}^{\prime}(y)\right)^{\prime}=\tilde{A}(y) \tilde{K}(y)\left(\frac{\tilde{K}(y)^{2}-\frac{1}{R^{2}}}{y^{2}}+\frac{1}{\gamma_{0}^{2}} \tilde{H}(y)^{2}\right.
\end{aligned}
$$




$$
\begin{aligned}
&\left.-\frac{1}{\gamma_{0}^{2}} \frac{\tilde{J}(y)^{2}}{\tilde{f}(y) \tilde{A}(y)^{2}}\right) \\
&\left(y^{2} \tilde{f}_{R}(y) \tilde{A}(y) \tilde{H}^{\prime}(y)\right)^{\prime}= 2 \tilde{A}(y) \tilde{H}(y) \tilde{K}(y)^{2} \\
& \tilde{f}_{R}(y) \tilde{A}(y)\left(\frac{y^{2} \tilde{J}^{\prime}(y)}{\tilde{A}(y)}\right)^{\prime}=2 \tilde{J}(y) \tilde{K}(y)^{2}
\end{aligned}
$$

where

$$
\begin{aligned}
& \tilde{V}_{1}=\gamma_{0}^{2} \tilde{K}^{\prime}(y)^{2}+\frac{y^{2}}{2} \tilde{H}^{\prime}(y)^{2} \\
& \tilde{V}_{2}=\gamma_{0}^{2} \frac{\left(\tilde{K}(y)^{2}-\frac{1}{R^{2}}\right)^{2}}{2 y^{2}}
\end{aligned}
$$

As explained above, our aim is to consider the case in which the gravitational equations decouple from the matter $\left(\kappa^{2} \rightarrow 0\right)$ leading to a background metric of the form (16). The relevant equations of motion for the gauge and Higgs field in the $R \rightarrow \infty$ limit are then

$$
\begin{gathered}
\left(\tilde{f}_{\infty}(y) \tilde{K}^{\prime}(y)\right)^{\prime}=\tilde{K}(y)\left(\frac{\tilde{K}(y)^{2}}{y^{2}}+\frac{1}{\gamma_{0}{ }^{2}} \tilde{H}(y)^{2}-\frac{1}{\gamma_{0}{ }^{2}} \frac{\tilde{J}(y)^{2}}{\tilde{f}_{\infty}(y)}\right) \\
\left(y^{2} \tilde{f}_{\infty}(y) \tilde{H}^{\prime}(y)\right)^{\prime}=2 \tilde{H}(y) \tilde{K}(y)^{2} \\
\tilde{f}_{\infty}(y)\left(y^{2} \tilde{J}^{\prime}(y)\right)^{\prime}=2 \tilde{J}(y) \tilde{K}(y)^{2} \\
\tilde{f}_{\infty}(y)=y^{2}-\frac{1}{y}
\end{gathered}
$$

Let us end this section by noting that the black hole temperature resulting from rescaling (21) and taking the $R \rightarrow \infty$ limit takes the form

$$
\hat{T} \equiv \frac{T}{e H_{0}}=\frac{3 \gamma_{0}}{4 \pi}
$$

\section{Holographic correspondence}

According to the AdS/CFT correspondence [1]-[3], properties of the dual 3 dimensional field theory defined on the boundary can be read from the behavior of the solution of the system in the bulk. In this approach temperature $\hat{T}$ in (531) corresponds to the temperature of the $d=3$ system. 
Let us first consider the vacuum expectation value in the $d=3$ field theory for the scalar operator $\mathcal{O}_{H}$, dual to the field $H$ defined on the bulk. It follows from the identification $\left\langle O_{H}\right\rangle \sim H_{1}$ with $H_{1}$ defined in eq.(27).

The asymptotic behavior of the $A_{0}$ component of the gauge field allows one to identify the chemical potential $\mu$ and the charge density $\rho$ of the 3 dimensional system. Indeed, given the ansatz for the $A_{0}$ component of the gauge field, eq.(12), the $U(1)$ electric field as defined in (34) reads

$$
E_{i}=\mathcal{F}_{i 0}^{U(1)}=\frac{H^{a}(x)}{H_{0}} F_{i 0}^{a}=e H_{0} H(x) \partial_{i} J(x)
$$

(the $\vec{H} \cdot \vec{A}_{0} \wedge \vec{A}_{i}$ contribution coming from the field strength commutator vanishes because $\vec{H} \| \vec{A}_{0}$ ).

Since $\lim _{x \rightarrow \infty} H(x)=1$, the electric field at infinity is given by

$$
\lim _{x \rightarrow \infty} E_{i}=e H_{0} \lim _{x \rightarrow \infty} \partial_{i} J(x)
$$

We have now to expand $J(x)$ for large $x$ and look for the $1 / x$ term which is the one leading to a nontrivial electric flux (i.e., leading to the dyon charge). The corresponding coefficient defines the charge density:

$$
\rho=-L H_{0} \tilde{J}_{1}
$$

Here and in what follow we use the coefficients of the asymptotic expansions defined in $\mathbb{R}^{2} \times S^{1}$. Following [18] we shall define a normal component $\rho_{n}$ by analyzing the expansion for $\tilde{J}(y)$ at the horizon using eq.(26),

$$
\rho_{n}=\tilde{j}_{h}
$$

and a superconducting charge density in the form

$$
\rho_{s}=\rho-\rho_{n}
$$

The constant value $\tilde{J}_{0}$ that $A_{\tau}$ takes at the boundary at infinity is related to the chemical potential through the formula

$$
\mu=H_{0} \tilde{J}_{0}
$$




\section{$5 \quad$ Numerical Analysis}

We shall restrict the numerical calculations to the case in which the boundary is $S^{1} \times \mathbb{R}^{2}$. We shall solve system (50)-(52) using the relaxation method [20] which determines the solution starting from an initial guess and improving it iteratively. The natural initial guess is the Prasad-Sommerfield solution [21] in flat space. Following this procedure, we have found regular solutions for different values of $\gamma_{0}$ i.e. of the temperature $\hat{T}$.

Figures 1 and 2 show a representative dyon solution that exists starting at the horizon. One can see that the scalar rapidly attains its symmetry breaking constant value while the magnetic and electric fields (associated to $K$ and $J$ respectively) concentrate in a spherical shell starting at the horizon. Figure 3 shows a remarkable property of the coupled system of non linear differential equations, namely the existence of several solutions satisfying the appropriate boundary conditions, each one corresponding to a different value of charge density $\rho$. This phenomenon was already encountered in [8] for the case of a $U(1)$ gauge field coupled to a complex scalar in an $\mathrm{AdS}_{4}$-Schwarzschild background. As it also happens in the $U(1)$ case, we have found that for increasing values of $\rho$ the solutions are distinguished by an increasing number of nodes $n$. Now, since evaluation of the free energy shows that it increases with the number of nodes (see below), we conclude that $n>1$ solutions are thermodynamically unfavorable and therefore we shall concentrate hecenforth on the zero node solution.
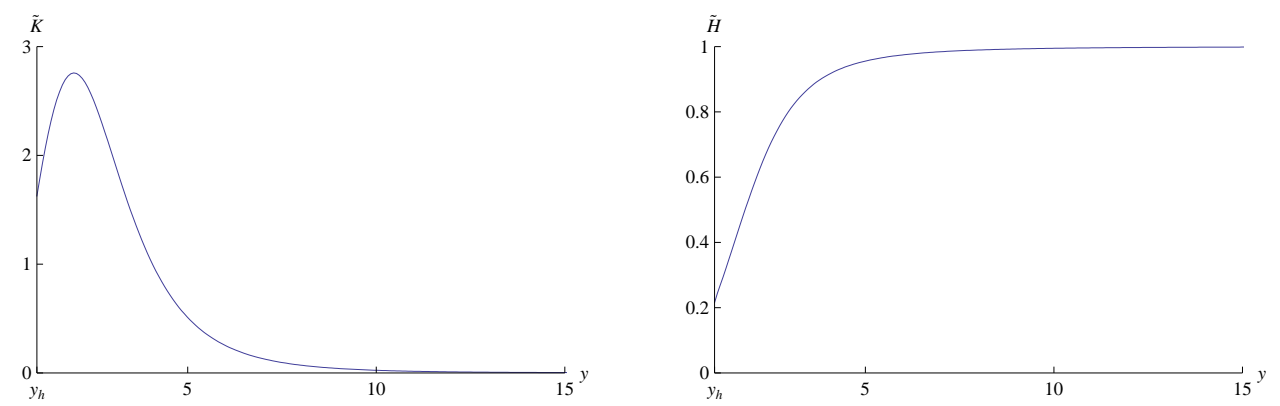

Figure 1: The solution for $\tilde{K}$ and $\tilde{H}$ as a function of $y$ or $\tilde{J}_{0}=6$ and $\gamma_{0}=0.2$ $\left(\hat{T}=3 \gamma_{0} /(4 \pi)\right)$. The solution exists starting at the horizon $y=y_{h}=1$. 


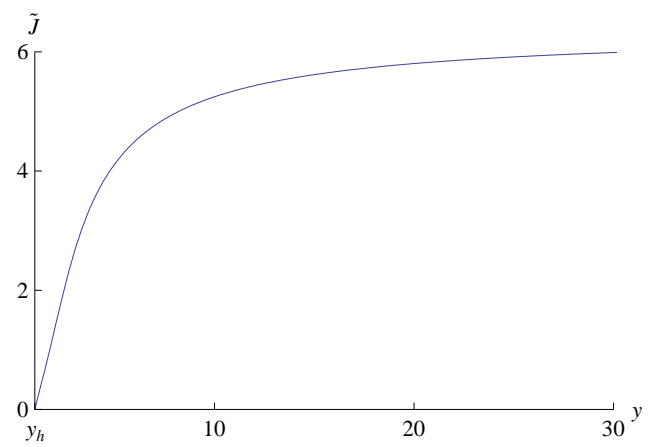

Figure 2: The solution for $\tilde{J}$ as a function of $y$ for $\tilde{J}_{0}=6$ and $\gamma_{0}=0.2$ $\left(\hat{T}=3 \gamma_{0} /(4 \pi)\right)$.

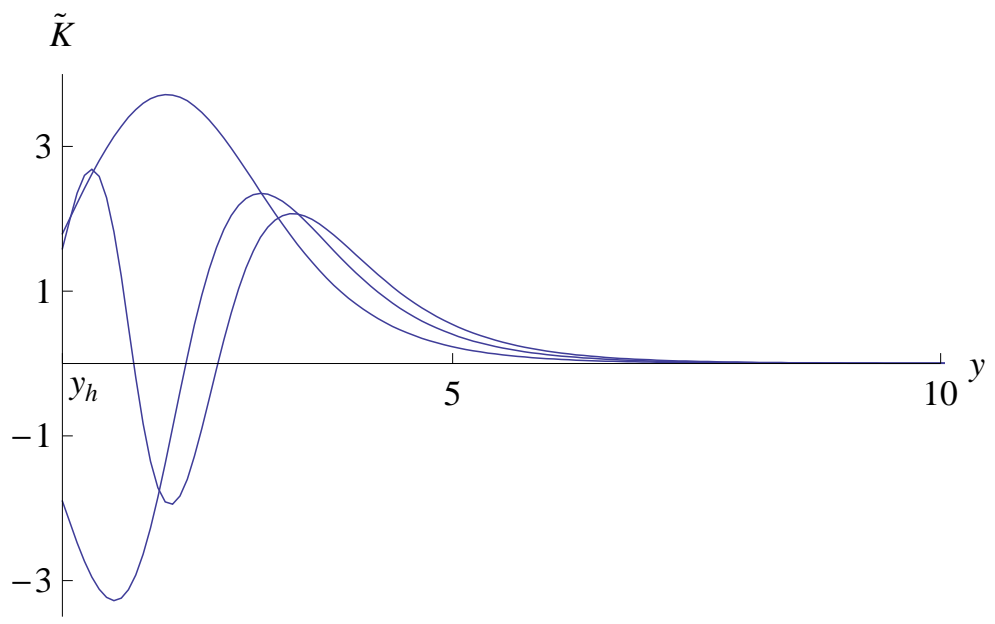

Figure 3: Different solutions for $\tilde{K}$ corresponding to different values of the charge density $\rho$. For increasing values of $\rho$ the solutions are distinguished by the increasing number of nodes.

The behavior of the condensate is shown in figure 4 where the asymptotic coefficient $H_{1} \sim\left\langle O_{H}\right\rangle$ in the asymptotic expansion (27) of the Higgs field is plotted as a function of the temperature (normalized in units of $e H_{0}$ ). In figures 5 and 6 we plot the coefficient $\tilde{K}_{1}$ in the asymptotic expansion of function $\tilde{K}$, while figure 7 shows the fraction $\rho_{s} / \rho$, as defined in eqs.(156)-(158) , related to the behavior of the electric field at the horizon and at infinity. From these figures we conclude that a finite temperature continuous symmetry breaking transition takes place so that the system condenses below a critical 
temperature $\hat{T}_{c}$. By fitting the curves we see that near $T_{c}$ one has a typical second order phase transition with power behavior of the form

$$
\begin{aligned}
\tilde{H}_{1} & \propto\left(\hat{T}_{c}-\hat{T}\right) \\
\tilde{K}_{1} & \propto\left(\hat{T}_{c}-\hat{T}\right)^{1 / 2} \quad \text { as } \hat{T} \rightarrow \hat{T}_{c} \\
\frac{\rho_{s}}{\rho} & \propto\left(\hat{T}_{c}-\hat{T}\right)
\end{aligned}
$$

Notice that $\tilde{K}_{1}$ has a critical exponent $1 / 2$ typical of second order transitions. One can see in figure 4 that at low temperature $\hat{H}_{1}$ appears to diverge. However, one should take into account that when the condensate becomes very large, the backreaction on the metric can no longer be neglected as we did by choosing a fixed Schwarzschild-AdS background metric.

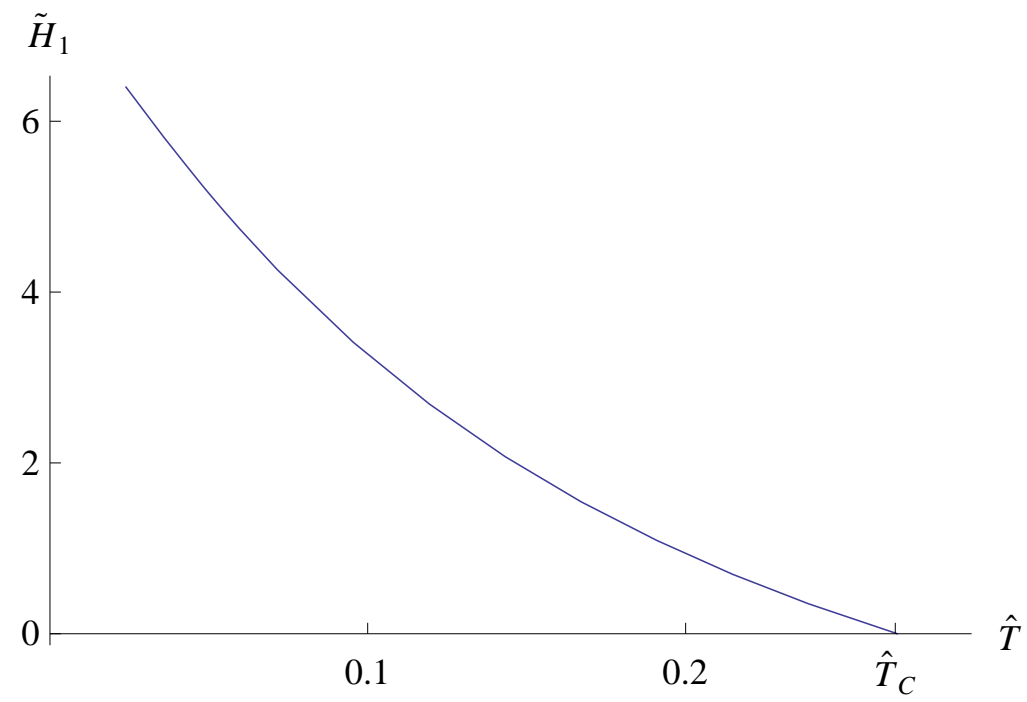

Figure 4: The $\tilde{H}_{1}$ coefficient in the Higgs field asymptotic expansion as a function of the temperature. We have taken $\tilde{J}_{0}=6$ and for this value the critical temperature is $\hat{T}_{c}=0.26649 \ldots$. 


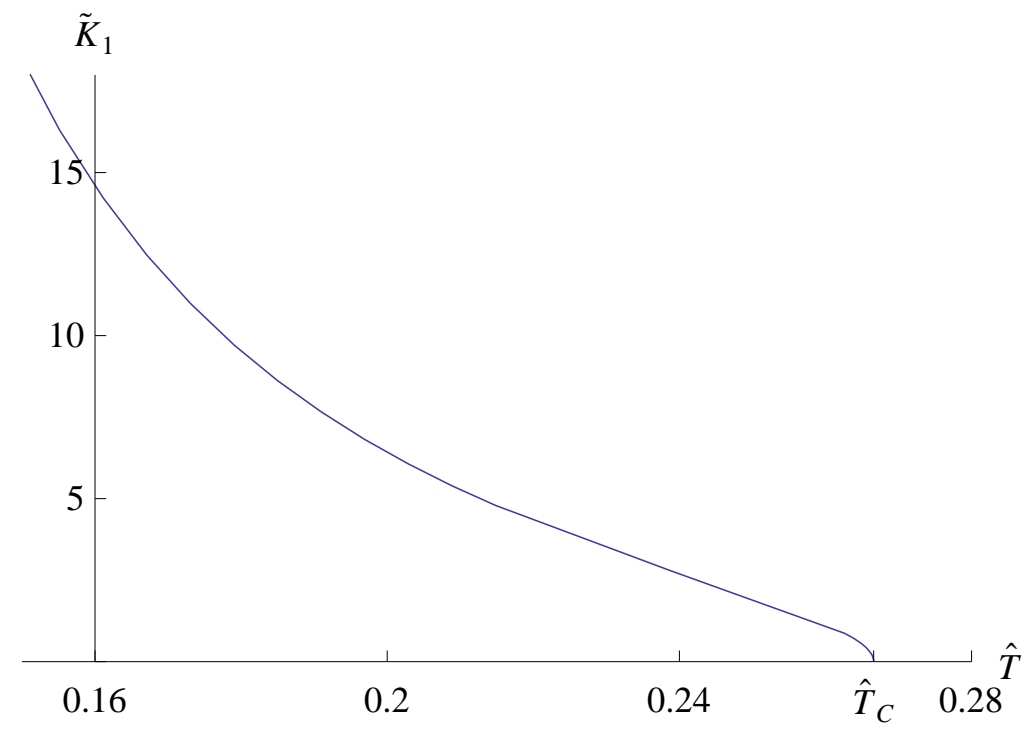

Figure 5: The $\tilde{K}_{1}$ coefficient in the gauge field asymptotic expansion as a function of the temperature for $\tilde{J}_{0}=6$.

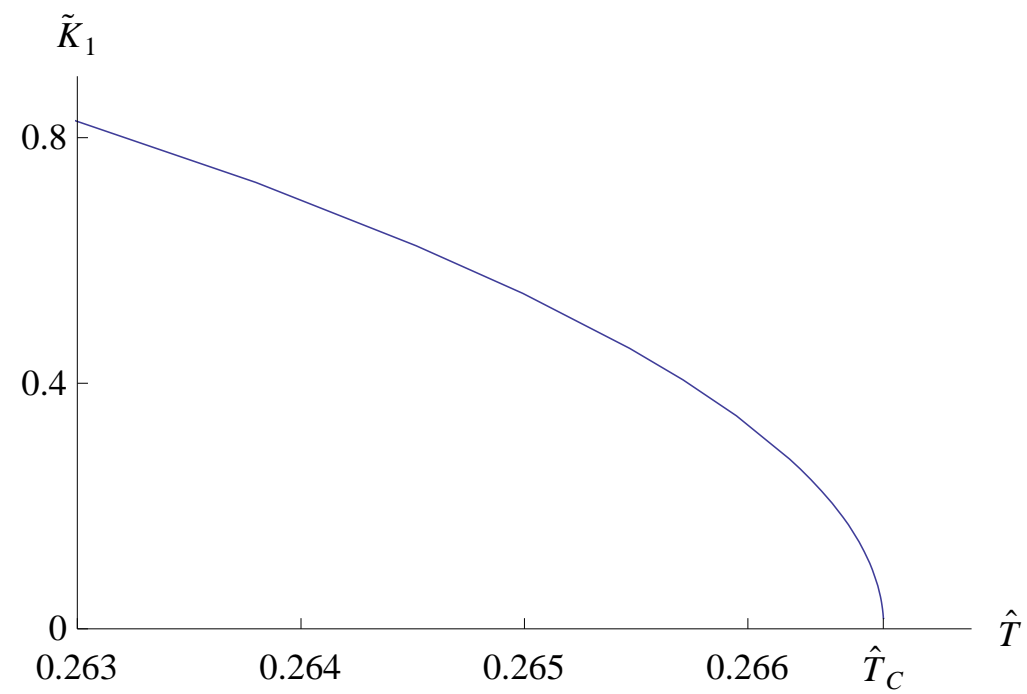

Figure 6: A zoomed view of $\tilde{K}_{1}$ near the critical point. One can see that its behavior near $T=T_{c}$ corresponds to $\sqrt{\hat{T}_{c}-\hat{T}}$. 


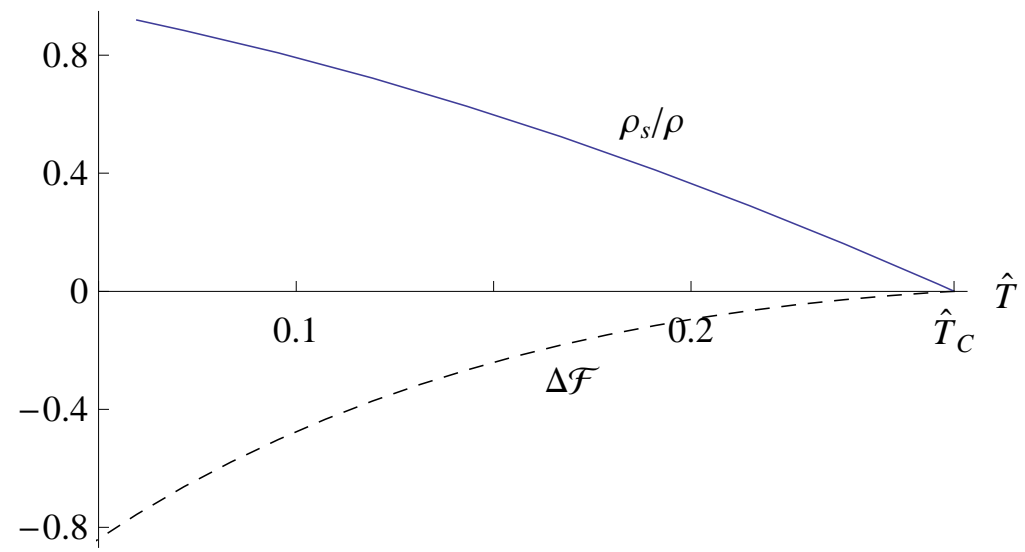

Figure 7: The solid line represents the fraction $\rho_{s} / \rho$ of the superconducting and the total charge densities. The dashed line the free energy difference between the condensed and the uncondensed phases. At $\hat{T}=\hat{T}_{c}, \rho_{s} / \rho$ vanishes linearly.

The free energy $\mathcal{F}$ is given by the on-shell action,

$$
\mathcal{F}=\left.T S_{E}\right|_{\text {on } \text { shell }}
$$

Using the Euclidean action as given in eq.(42) we have numerically computed $\mathcal{F}$ for the dyon solution and compared it with the free energy in the uncondensed phase which corresponds to the solution $\tilde{H}(y)=1, \tilde{K}(y)=$ $0, \tilde{J}(y)=\tilde{J}_{0}(1-1 / y)$. We plot in figure 7 the free energy density difference $\Delta \mathcal{F}$, normalized in units of $H_{0}^{2} /(2 L)$, which can be seen to be continuous at $\hat{T}=\hat{T}_{c}$.

\section{Summary and discussion}

We have applied the AdS/CFT correspondence to describe the strong coupling regime of a $2+1$ quantum field theory which undergoes a phase transition exhibiting the condensation of a composite charge operator below a critical temperature $T_{c}$. The dual gravity theory consists of an $S U(2)$ YangMills-Higgs model in a Schwarzschild-AdS 4 black hole with temperature $T$.

Breaking the gauge symmetry down to $U(1)$ we started by constructing a spherically symmetric soliton solution in the bulk, having both magnetic and electric charge. By choosing appropriate conditions at the horizon and 
at infinity, we found a family of dyon solutions leading to the existence of a condensate, below a critical temperature, in the dual field theory on the boundary which, after an appropriate scaling, becomes $\mathbb{R}^{2} \times S^{1}$.

By calculating the free energy $\mathcal{F}$ we have seen that the condensed phase is thermodynamically favored and we were able to determine which one of the dyon family solutions had the lowest $\mathcal{F}$-value. We have also calculated the power behavior of the condensate, typical of a second order phase transition.

There are many issues that we have not discussed in our work and that deserve a thorough study. To begin with, one should consider the applicability of our results to describe the behavior of an actual condensed matter system. Also, since we have found on the gravity side classical solutions with quantized magnetic flux, we expect that the issue of confinement through the appearence of chromoelectric flux tubes in the dual strongly coupled QFT could take place. In this respect, it should be of interest to consider monopole solutions in Schwarzschild- $\mathrm{AdS}_{5}$ black hole backgrounds so that the dual theory is defined in three spatial dimensions. We hope to discuss theses issues in a future publication.

Acknowledgments We would like to thank Jorge Russo for discussions and helpful suggestions and Tameem Albash for a clarifying comment. A.R.L. is grateful with Borut Bajc for helpful discussions. This work was partially

supported by PIP6160-CONICET, BID 1728OC/AR PICT20204-ANPCYT grants and by CIC and UNLP, Argentina.

\section{References}

[1] J. M. Maldacena, "The large N limit of superconformal field theories and supergravity," Adv. Theor. Math. Phys. 2 (1998) 231 [Int. J. Theor. Phys. 38 (1999) 1113] [arXiv:hep-th/9711200].

[2] S. S. Gubser, I. R. Klebanov and A. M. Polyakov, "Gauge theory correlators from non-critical string theory," Phys. Lett. B 428 (1998) 105 arXiv:hep-th/9802109].

[3] E. Witten, Adv. Theor. Math. Phys. 2 (1998) 253 arXiv:hep-th/9802150]. 
[4] S. S. Gubser, "Phase transitions near black hole horizons," Class. Quant. Grav. 22 (2005) 5121 arXiv:hep-th/0505189].

[5] S. S. Gubser, "Breaking an Abelian gauge symmetry near a black hole horizon," Phys. Rev. D 78 (2008) 065034 [arXiv:0801.2977 [hep-th]].

[6] S. A. Hartnoll, C. P. Herzog and G. T. Horowitz, "Building a Holographic Superconductor," Phys. Rev. Lett. 101 (2008) 031601 arXiv:0803.3295 [hep-th]].

[7] S. S. Gubser, "Colorful horizons with charge in anti-de Sitter space," Phys. Rev. Lett. 101 (2008) 191601 [arXiv:0803.3483 [hep-th]].

[8] T. Albash and C. V. Johnson, "Phases of Holographic Superconductors in an External Magnetic Field," arXiv:0906.0519 [hep-th]; "Vortex and Droplet Engineering in Holographic Superconductors," arXiv:0906.1795 [hep-th].

[9] M. Montull, A. Pomarol and P. J. Silva, "The Holographic Superconductor Vortex," Phys. Rev. Lett. 103 (2009) 091601 arXiv:0906.2396 [hep-th]].

[10] S. S. Gubser and S. S. Pufu, JHEP 0811 (2008) 033 arXiv:0805.2960 [hep-th]].

[11] R. Manvelyan, E. Radu and D. H. Tchrakian, Phys. Lett. B 677 (2009) 79 arXiv:0812.3531 [hep-th]]; S. Franco, A. Garcia-Garcia and D. Rodriguez-Gomez, arXiv:0906.1214 and 0911.1354 [hep-th]; K. Peeters, J. Powell and M. Zamaklar, JHEP 0909 (2009) 101 arXiv:0907.1508 [hep-th]]; K. Goldstein, S. Kachru, S. Prakash and S. P. Trivedi, arXiv:0911.3586 [hep-th]; F. Aprile and J. G. Russo, arXiv:0912.0480 [hep-th]; K. Maeda, M. Natsuume and T. Okamura, "Vortex lattice for a holographic superconductor," Phys. Rev. D 81 (2010) 026002 arXiv:0910.4475 [hep-th]; M. Cadoni, G. D'Appollonio and P. Pani, "Phase transitions between Reissner-Nordstrom and dilatonic black holes in 4D AdS spacetime," arXiv:0912.3520 [hep-th]; D. Bak and S. J. Rey, "Composite Fermion Metals from Dyon Black Holes and S-Duality," arXiv:0912.0939 [hep-th].

[12] A. R. Lugo and F. A, Schaposnik, Phys. Lett. B 467 (1999)43. 
[13] A. R. Lugo, E. F. Moreno and F. A, Schaposnik, Phys. Lett. B 473 (2000)35.

[14] G.W. Gibbons, "Self gravitating magnetic monopoles, global monopoles and black holes", lectures given at 12th Lisbon Autumn School on Physics, Lisbon, Portugal, Oct 1-5, 1990. Published in Lisbon Autumn School 1990:110-133.

[15] A. M. Polyakov, "Particle spectrum in quantum field theory," JETP Lett. 20 (1974) 194 [Pisma Zh. Eksp. Teor. Fiz. 20 (1974) 430].

[16] S. W. Hawking and D. N. Page, "Thermodynamics Of Black Holes In Anti-De Sitter Space," Commun. Math. Phys. 87, 577 (1983).

[17] E. Witten, "Anti-de Sitter space, thermal phase transition, and confinement in gauge theories," Adv. Theor. Math. Phys. 2 (1998) 505

[18] S. S. Gubser and S. S. Pufu, "The gravity dual of a p-wave superconductor," JHEP 0811 (2008) 033 arXiv:0805.2960 [hep-th]].

[19] B. Julia and A. Zee, "Poles With Both Magnetic And Electric Charges In Nonabelian Gauge Theory," Phys. Rev. D 11 (1975) 2227.

[20] W. H. Press, S. A. Teukolsky, W. V. Vetterlink, Numerical Recipes: The art of Scientific Computing, Cambridge University Press, Cambridge UK, 1992.

[21] M. K. Prasad and C. M. Sommerfield, Phys. Rev. Lett. 35 (1975) 760. 


\title{
Holographic phase transition from dyons in an AdS black hole background
}

\author{
A. R. Lugo ${ }^{a *}$ E. F. Moreno ${ }^{b}$ and F. A. Schaposnik ${ }^{a \dagger}$ \\ ${ }^{a}$ Departamento de Física-IFLP, Universidad Nacional de La Plata \\ C.C. 67, 1900 La Plata, Argentina \\ ${ }^{b}$ Department of Physics, West Virginia University \\ Morgantown, West Virginia 26506-6315, U.S.A.
}

October 29, 2018

\begin{abstract}
We construct a dyon solution for a Yang-Mills-Higgs theory in a 4 dimensional Schwarzschild-anti-de Sitter black hole background with temperature T. We then apply the AdS/CFT correspondence to describe the strong coupling regime of a $2+1$ quantum field theory which undergoes a phase transition exhibiting the condensation of a composite charge operator below a critical temperature $T_{c}$.
\end{abstract}

\section{Introduction}

It has recently been observed that the AdS/CFT correspondence [1]-[3] provides a powerful tool to study quantum critical dynamics in condensed matter models for superconductivity, superfluidity and Hall effect [4]-[10]. In this way, using the dual classical gravity description, correlation functions in strongly interacting systems can be calculated and the relevant physical

${ }^{*}$ Associated with CONICET

${ }^{\dagger}$ Associated with CICBA 
properties can be determined in a relatively simple way, which has motivated an intense activity in the field (some works related to the present approach are listed in [1] ).

Temperature is introduced in the gravity dual through the presence of a Schwarzschild-AdS black hole, either added as a background or resulting from back reaction. Apart from gauge fields, the models referred above include minimally coupled scalar matter fields. In the Abelian case the ansatz for gauge fields included an electrostatic potential $A_{0}$ which is spatially independent at the boundary as also is the case for the scalar. Spatially dependent droplet and vortex solutions have been also studied in [8]-[9] and in the nonAbelian case non-trivial gauge-field components were considered [7],[10]. In general, such classical solutions in the bulk exist below a certain critical temperature leading to thermodynamically favored phases in the the strongly coupled quantum field theory on the boundary.

Following the ideas described above, we consider in the present paper a model not yet studied: a dyon solutions for Yang-Mills-Higgs theory in a 4 dimensional Schwarzschild-anti-de Sitter black-hole background metric. We shall be guided by our previous work on monopole and dyon solutions in $\mathrm{AdS}_{4}$ space [12]-[13] where, due to the absence of singularities, the spherically symmetric solutions were constructed in the whole radial domain $\mathbb{R}^{+}$, while in the present case the radial variable extend from the black hole horizon to infinity. The black hole temperature, determined by the horizon, will become the temperature of the field theory defined on the boundary. The matter field will lead to the non-Abelian gauge symmetry breaking condensate while the field strength associated to the surviving $U(1)$ symmetry will correspond to that of a dyon with electric and magnetic charges.

The paper is organized as follows. In section 2 we present the gravityYang-Mills-Higgs model and specialize to the case in which the gravitational equations decouple from matter leading to an AdS-Schwarzschild black hole background for the gauge-matter system. Then in section 3 we analyze the properties of the dyon solution considering both the cases of an $S^{2} \times S^{1}$ and $\mathbb{R}^{2} \times S^{1}$ boundaries. In section 4 we proceed to describe the holography calculation of relevant quantities in the dual field theory defined on the boundary (taken as $\mathbb{R}^{2} \times S^{1}$ ) and present our numerical results. We end with a summary and discussion in section 5 . 


\section{The gravity-Yang-Mills-Higgs system}

\subsection{The model}

We consider a gravity-Yang-Mills-Higgs system with gauge group $S U(2)$ and the scalar field in the adjoint representation, in a 4 dimensional space-time with Minkowski signature $(-,+,+,+)$. The action takes the form

$$
S=S_{G}+S_{Y M}+S_{H}=\int d^{4} x \sqrt{|g|}\left(L_{G}+L_{Y M}+L_{H}\right)
$$

with

$$
\begin{gathered}
L_{G}=\frac{1}{2 \kappa^{2}}(R-2 \Lambda) \\
L_{Y M}=-\frac{1}{4 e^{2}} F_{\mu \nu}^{a} F^{a \mu \nu} \\
L_{H}=-\frac{1}{2} D_{\mu} H^{a} D^{\mu} H^{a}-V(H) \\
V(H)=\frac{\lambda}{4}\left(H^{a} H^{a}-H_{0}^{2}\right)^{2} \\
\kappa^{2} \equiv 8 \pi G_{N}
\end{gathered}
$$

Here $G_{N}$ is the Newton constant, $e$ the gauge coupling and $\Lambda$ the cosmological constant (with our conventions $\Lambda<0$ corresponds, in the absence of matter, to anti-de Sitter space). The field strength $F_{\mu \nu}^{a}(a=1,2,3)$ is defined as

$$
F_{\mu \nu}^{a}=\partial_{\mu} A_{\nu}^{a}-\partial_{\nu} A_{\mu}^{a}+\varepsilon^{a b c} A_{\mu}^{b} A_{\nu}^{c}
$$

and the covariant derivative $D_{\mu}$ acting on the Higgs triplet $H^{a}$ is given by

$$
D_{\mu} H^{a}=\partial_{\mu} H^{a}+\varepsilon^{a b c} A_{\mu}^{b} H^{c}
$$

The equations of motion that follow from (11) are

$$
\begin{aligned}
E_{\mu \nu}+\Lambda g_{\mu \nu} & =\kappa^{2}\left(T_{\mu \nu}^{Y M}+T_{\mu \nu}^{H}\right) \\
D_{\rho} D^{\rho} H^{a} & =\frac{\delta V(H)}{\delta H^{a}} \\
\frac{1}{e^{2}} D^{\rho} F_{\mu \rho}^{a} & =\varepsilon^{a b c}\left(D_{\mu} H^{b}\right) H^{c}
\end{aligned}
$$


where $E_{\mu \nu}$ is the Einstein tensor and the matter energy-momentum tensor $T_{\mu \nu}$,

$$
T_{\mu \nu}=-2 \frac{\delta S}{\delta g^{\mu \nu}}
$$

is given by

$$
\begin{aligned}
T_{\mu \nu}^{Y M} & =\frac{1}{e^{2}} F_{\mu \rho}^{a} F_{\nu}^{a \rho}+g_{\mu \nu} L_{Y M} \\
T_{\mu \nu}^{H} & =D_{\mu} H^{a} D_{\nu} H^{a}+g_{\mu \nu} L_{H}
\end{aligned}
$$

The most general static spherically symmetric form for the metric in 3 spatial dimensions together with the t'Hooft-Polyakov-Julia-Zee ansatz for the gauge and Higgs fields in the usual vector notation reads [12]-[13]

$$
\begin{aligned}
g & =-\mu(x) A(x)^{2} d^{2} t+\mu(x)^{-1} d^{2} r+r^{2} d^{2} \Omega_{2} \\
\vec{A} & =d t e H_{0} J(x) \check{e}_{r}-d \theta(1-K(x)) \check{e}_{\varphi}+d \varphi(1-K(x)) \sin \theta \check{e}_{\theta} \\
\vec{H} & =H_{0} H(x) \check{e}_{r}
\end{aligned}
$$

where we have introduced the dimensionless radial coordinate $x \equiv e H_{0} r$ and we denote the standard spherical unit vectors as $\check{e}_{r}, \check{e}_{\varphi}, \check{e}_{\theta}$. $H_{0}$ sets the mass scale $\left(\left[H_{0}\right]=m^{1}\right)$.

Using this ansatz, the equations of motion take the form

$$
\begin{aligned}
& (x \mu(x))^{\prime}=1+3 \gamma_{0}^{2} x^{2}-\kappa^{2} H_{0}^{2}\left(\mu(x) V_{1}+V_{2}+\frac{x^{2}}{2} \frac{J^{\prime}(x)^{2}}{A(x)^{2}}\right. \\
& \left.+\frac{J(x)^{2} K(x)^{2}}{\mu(x) A(x)^{2}}\right) \\
& x A^{\prime}(x)=\kappa^{2} H_{0}^{2}\left(V_{1}+\frac{J(x)^{2} K(x)^{2}}{\mu(x)^{2} A(x)^{2}}\right) A(x) \\
& \left(\mu(x) A(x) K^{\prime}(x)\right)^{\prime}=A(x) K(x)\left(\frac{K(x)^{2}-1}{x^{2}}+H(x)^{2}\right. \\
& \left.-\frac{J(x)^{2}}{\mu(x) A(x)^{2}}\right) \\
& \mu(x) A(x)\left(\frac{x^{2} J^{\prime}(x)}{A(x)}\right)^{\prime}=2 J(x) K(x)^{2} \\
& \left(x^{2} \mu(x) A(x) H^{\prime}(x)\right)^{\prime}=A(x) H(x)\left(2 K(x)^{2}+\frac{\lambda}{e^{2}} x^{2}\left(H(x)^{2}-1\right)\right)
\end{aligned}
$$


where, for convenience, we have defined the dimensionless parameter

$$
\gamma_{0}^{2} \equiv-\frac{\Lambda}{3 e^{2} H_{0}^{2}}=\frac{1}{L^{2} e^{2} H_{0}^{2}} \quad, \quad L^{2} \equiv-\frac{3}{\Lambda}
$$

and

$$
\begin{aligned}
& V_{1}=K^{\prime}(x)^{2}+\frac{x^{2}}{2} H^{\prime}(x)^{2} \\
& V_{2}=\frac{\left(K(x)^{2}-1\right)^{2}}{2 x^{2}}+\frac{\lambda}{4 e^{2}} x^{2}\left(H(x)^{2}-1\right)^{2}
\end{aligned}
$$

\subsection{The AdS-Schwarzschild black hole background}

In the $\kappa^{2} \rightarrow 0$ limit the gravitational equations in system (13) decouple from the matter ones leading to a solution of the metric $g$ of the form

$$
g=-\mu_{0}(x) d^{2} t+\mu_{0}^{-1}(x) d^{2} r+r^{2} d^{2} \Omega_{2}
$$

with

$$
\mu_{0}(x)=1+\gamma_{0}^{2} x^{2}-\frac{R^{3}}{\gamma_{0} x}
$$

Here $R$ is a dimensionless parameter related to the black hole mass. For $R \neq 0$ the metric defined in (16) has an horizon at $x=x_{h}$, i.e.,

$$
\mu_{0}\left(x_{h}\right)=0
$$

with $\mu_{0}^{\prime}\left(x_{h}\right) \neq 0$. Written in terms of the parameters of the model $x_{h}$ reads

$$
\gamma_{0} x_{h}=\left(\sqrt{\frac{R^{6}}{4}+\frac{1}{27}}+\frac{R^{3}}{2}\right)^{1 / 3}-\left(\sqrt{\frac{R^{6}}{4}+\frac{1}{27}}-\frac{R^{3}}{2}\right)^{1 / 3}
$$

In order to compute the black hole temperature one uses the standard recipe [16] leading to no conical singularity at the horizon after Wick rotation to the Euclidean time $\tau_{E} \equiv i t$. Hence, given (17) and imposing

$$
\tau_{E} \sim \tau_{E}+\beta
$$

one identifies the black hole temperature as

$$
T \equiv \frac{1}{\beta}=\frac{\left|\mu_{0}^{\prime}\left(x_{h}\right)\right| e H_{0}}{4 \pi}
$$




\section{The dyon in the black hole background}

\subsection{Properties of the solution}

From here on we shall consider, for simplicity, the BPS limit of the potential (5) which correspond to $\lambda / e^{2}=0$ with $H_{0}$ fixed. Taking the black hole metric (17) as a background we are left with the system

$$
\begin{aligned}
& \left(\mu_{0}(x) K^{\prime}(x)\right)^{\prime}=K(x)\left(\frac{K(x)^{2}-1}{x^{2}}+H(x)^{2}-\frac{J(x)^{2}}{\mu_{0}(x)}\right) \\
& \mu_{0}(x)\left(x^{2} J^{\prime}(x)\right)^{\prime}=2 J(x) K(x)^{2} \\
& \left(x^{2} \mu_{0}(x) H^{\prime}(x)\right)^{\prime}=2 H(x) K(x)^{2}
\end{aligned}
$$

We shall look for a solution to (22) $-(24)$ which is regular at the horizon

$$
K(x), H(x), J(x) /\left(x-x_{h}\right) \quad \text { regular at } x_{h}
$$

so that

$$
\begin{aligned}
H(x) & =h_{0}+h_{1}\left(x-x_{h}\right)+\mathcal{O}\left[\left(x-x_{h}\right)^{2}\right] \\
K(x) & =k_{0}+k_{1}\left(x-x_{h}\right)+\mathcal{O}\left[\left(x-x_{h}\right)^{2}\right] \\
J(x) & =j_{h}\left(x-x_{h}\right)+\mathcal{O}\left[\left(x-x_{h}\right)^{2}\right]
\end{aligned}
$$

Concerning $x \rightarrow \infty$, the asymptotic behavior takes the form

$$
\begin{array}{rlr}
|\vec{H}(x)| & =H_{0}\left(1-\frac{H_{1}}{x^{3}}+\frac{H_{2}}{x^{5}}+\frac{H_{3}}{x^{4+2 \nu}}+\cdots\right) \\
K(x) & =\frac{K_{1}}{x^{\nu+1}}+\frac{K_{3}}{x^{\nu+3}},+\cdots \quad \nu \in \mathbb{R} \quad \text { as } \mathrm{x} \rightarrow \infty \\
J(x) & =J_{0}+\frac{J_{1}}{x}+\ldots &
\end{array}
$$

Such behavior is consistent with the equation of motion (22) provided that the following condition holds

$$
\frac{1}{\gamma_{0}^{2}}=\nu(\nu+1)
$$

Equation (30) has two solutions,

$$
\nu_{ \pm}=-\frac{1}{2} \pm \frac{1}{2} \sqrt{1+\frac{4}{\gamma_{0}^{2}}}
$$


Only the $\nu_{+}$root gives an acceptable asymptotic behavior for $K(x)$ so that

$$
K(x) \sim \frac{k_{\nu}}{x^{\nu++1}}=\frac{k_{\nu}}{x^{\frac{1}{2}\left(1+\sqrt{1+\frac{4}{\gamma_{0}^{2}}}\right)}} \quad \text { as } x \rightarrow \infty
$$

Being the $S U(2)$ gauge symmetry of the Lagrangian spontaneously broken by the expectation value of the scalar field, we shall define the field strength associated with the surviving $U(1)$ symmetry as in [15]

$$
\mathcal{F}_{\mu \nu}^{U(1)} \equiv \frac{H^{a}}{H_{0}} F_{\mu \nu}^{a}
$$

From this expression one finds the U(1) magnetic and electric fields

$$
B^{i}=\frac{1}{2} \frac{\varepsilon^{i j k}}{\sqrt{g^{(3)}}} \mathcal{F}_{j k}^{U(1)}, \quad E_{i}=\mathcal{F}_{i 0}^{U(1)}
$$

and the magnetic and electric charges

$$
\begin{aligned}
Q_{m} & =\int_{V} d^{3} x \sqrt{g^{(3)}} \nabla_{i}^{(3)} B^{i} \\
Q_{e} & =\int_{V} d^{3} x \sqrt{g^{(3)}} \nabla_{i}^{(3)} E^{i}
\end{aligned}
$$

where $\nabla_{i}^{(3)}$ is the 3-dimensional covariant derivative. In order to compute $Q_{m}$ one just needs the asymptotic value of $\mathcal{F}_{i j}^{U(1)}$ since eqs.(34)-(35) imply

$$
Q_{m}=\frac{1}{2} \int_{\partial V=S^{2}} \varepsilon^{i j k} \mathcal{F}_{j k}^{U(1)} d S_{i}
$$

Now, from the asymptotic conditions (29) one has

$$
\varepsilon^{i j k} \mathcal{F}_{j k}^{U(1)} \sim \frac{1}{x^{2}} \check{e}_{r} \delta^{i r} \quad \text { for } x \rightarrow \infty
$$

and then

$$
Q_{m}=-\frac{1}{e} \int_{S^{2}} d \Omega=-\frac{4 \pi}{e}
$$

As expected, the spherically symmetric solution has a quantized $n=-1$ magnetic charge (in units of $4 \pi / e$ ). 
Concerning the electric charge, one can see from the asymptotic behavior of $J$, eq.(29), that $J_{0}$ which sets the charge scale and $J_{1}$, determines its value. One can write

$$
Q_{e}=\frac{1}{e} \mathcal{Q}\left(e^{2} / \lambda, J_{0}\right)
$$

with $\mathcal{Q}$ a dimensionless function to be determined numerically. As for the Julia-Zee flat space dyon, the electric charge is not quantized at the classical level. As we shall see, $J_{0}$ and $J_{1}$ will be identified, through holography, with the chemical potential and charge density of the strongly coupled system respectively.

Let us now find the expression for the dyon action which will be connected with the free energy of the field theory defined on the boundary. Inserting ansatz (12) in eqs.(33)-(4) one obtains for the Yang-Mills and Higgs Lagrangians

$$
\begin{aligned}
L_{Y M} & =-\frac{e^{2} H_{0}^{4}}{2}\left(-\frac{J^{\prime 2}}{A^{2}}-2 \frac{J^{2} K^{2}}{x^{2} \mu A^{2}}+2 \frac{\mu K^{\prime 2}}{x^{2}}+\frac{\left(K^{2}-1\right)^{2}}{x^{4}}\right) \\
L_{H} & =-\frac{e^{2} H_{0}^{4}}{2}\left(\mu H^{\prime 2}+2 \frac{K^{2} H^{2}}{x^{2}}\right)
\end{aligned}
$$

Then, by analytic continuation, the Euclidean action takes the form

$$
S_{E}=-\int_{0}^{\beta} d \tau_{E} \int_{V} d^{3} x \sqrt{g}\left(L_{Y M}+L_{H}\right)
$$

\subsection{From $\mathrm{S}^{2}$ to $\mathbb{R}^{2}$}

As stated in the introduction, our aim is to calculate the thermodynamics of the strongly interacting field theory defined on the boundary $\partial M$ using the dual classical description of the gravity system governed by the action (11) in the 4 dimensional manifold $M$; now, according to (16), $\partial M=S^{2} \times$ $S^{1}$. From the condensed matter viewpoint, it is more relevant to study the finite temperature field theory defined on the plane $\mathbb{R}^{2}$ and not on the sphere $S^{2}$. To this end, one can follow the approach in [17] and proceed to a change coordinates $(t, x, \theta, \varphi) \rightarrow\left(\tau, y, x^{1}, x^{2}\right)$ or, equivalently, $(t, x, \theta, \varphi) \rightarrow$ $(\tau, y, \rho, \varphi)$

$$
\begin{aligned}
\tau & =\underset{R}{R} t \\
y & =\frac{\gamma_{0}}{R} x
\end{aligned}
$$




$$
\begin{aligned}
& x^{1}=2 R \tan \frac{\theta}{2} \cos \varphi=\rho \cos \varphi \\
& x^{2}=2 R \tan \frac{\theta}{2} \sin \varphi=\rho \sin \varphi
\end{aligned}
$$

together with the field redefinitions

$$
\begin{aligned}
\tilde{A}(y) & =A(x) \\
\tilde{\xi}(y) & =R^{-2} \xi(x) \\
\tilde{H}(y) & =H(x) \\
\tilde{K}(y) & =R^{-1} K(x) \\
\tilde{J}(y) & =R^{-1} J(x)
\end{aligned}
$$

where $R$ was introduced in (17). After this change, ansatz (12) becomes

$$
\begin{aligned}
g & =-\tilde{f}_{R}(y) \tilde{A}(y)^{2} d^{2} \tau+L^{2} \frac{d^{2} y}{\tilde{f}_{R}(y)}+L^{2} y^{2} \frac{d^{2} \vec{x}}{\left(1+\frac{\rho^{2}}{4 R^{2}}\right)^{2}} \\
\vec{A} & =d \tau \text { e } H_{0} \tilde{J}(y)\left(\frac{4 R \rho}{\rho^{2}+4 R^{2}} \check{e}_{\rho}+\frac{4 R^{2}-\rho^{2}}{\rho^{2}+4 R^{2}} \check{e}_{3}\right)^{2} \\
& +\frac{4 R^{2}}{\rho^{2}+4 R^{2}}\left(\frac{1}{R}-\tilde{K}(y)\right)\left[\left(\frac{4 R^{2}-\rho^{2}}{\rho^{2}+4 R^{2}} \check{e}_{\rho}-\frac{4 R \rho}{\rho^{2}+4 R^{2}} \check{e}_{3}\right) \rho d \varphi\right. \\
& \left.-\check{e}_{\varphi} d \rho\right] \\
\vec{H} & =H_{0} \tilde{H}(y)\left(\frac{4 R \rho}{\rho^{2}+4 R^{2}} \check{e}_{\rho}+\frac{4 R^{2}-\rho^{2}}{\rho^{2}+4 R^{2}} \check{e}_{3}\right)
\end{aligned}
$$

where

$$
\tilde{f}_{R}(y)=f_{R}(y)+\tilde{\xi}(y) \quad, \quad f_{R}(y) \equiv \frac{1}{R^{2}}+y^{2}-\frac{1}{y}
$$

and the equations of motion for the Einstein-Yang Mills-Higgs system, eqs. (13) take the form,

$$
\begin{aligned}
& (y \tilde{\xi}(y))^{\prime}=-\kappa^{2} H_{0}{ }^{2}\left(\tilde{f}_{R}(y) \tilde{V}_{1}+\tilde{V}_{2}+\frac{y^{2}}{2} \frac{\tilde{J}^{\prime}(y)^{2}}{\tilde{A}(y)^{2}}+\frac{\tilde{J}(y)^{2} \tilde{K}(y)^{2}}{\tilde{f}_{R}(y) \tilde{A}(y)^{2}}\right) \\
& y \tilde{A}^{\prime}(y)=\kappa^{2} H_{0}{ }^{2}\left(\tilde{V}_{1}+\frac{\tilde{J}(y)^{2} \tilde{K}(y)^{2}}{\tilde{f}_{R}(y)^{2} \tilde{A}(y)^{2}}\right) \tilde{A}(y) \\
& \left(\tilde{f}_{R}(y) \tilde{A}(y) \tilde{K}^{\prime}(y)\right)^{\prime}=\tilde{A}(y) \tilde{K}(y)\left(\frac{\tilde{K}(y)^{2}-\frac{1}{R^{2}}}{y^{2}}+\frac{1}{\gamma_{0}^{2}} \tilde{H}(y)^{2}\right.
\end{aligned}
$$




$$
\begin{aligned}
&\left.-\frac{1}{\gamma_{0}^{2}} \frac{\tilde{J}(y)^{2}}{\tilde{f}(y) \tilde{A}(y)^{2}}\right) \\
&\left(y^{2} \tilde{f}_{R}(y) \tilde{A}(y) \tilde{H}^{\prime}(y)\right)^{\prime}= 2 \tilde{A}(y) \tilde{H}(y) \tilde{K}(y)^{2} \\
& \tilde{f}_{R}(y) \tilde{A}(y)\left(\frac{y^{2} \tilde{J}^{\prime}(y)}{\tilde{A}(y)}\right)^{\prime}=2 \tilde{J}(y) \tilde{K}(y)^{2}
\end{aligned}
$$

where

$$
\begin{aligned}
& \tilde{V}_{1}=\gamma_{0}^{2} \tilde{K}^{\prime}(y)^{2}+\frac{y^{2}}{2} \tilde{H}^{\prime}(y)^{2} \\
& \tilde{V}_{2}=\gamma_{0}^{2} \frac{\left(\tilde{K}(y)^{2}-\frac{1}{R^{2}}\right)^{2}}{2 y^{2}}
\end{aligned}
$$

As explained above, our aim is to consider the case in which the gravitational equations decouple from the matter $\left(\kappa^{2} \rightarrow 0\right)$ leading to a background metric of the form (16). The relevant equations of motion for the gauge and Higgs field in the $R \rightarrow \infty$ limit are then

$$
\begin{gathered}
\left(\tilde{f}_{\infty}(y) \tilde{K}^{\prime}(y)\right)^{\prime}=\tilde{K}(y)\left(\frac{\tilde{K}(y)^{2}}{y^{2}}+\frac{1}{\gamma_{0}{ }^{2}} \tilde{H}(y)^{2}-\frac{1}{\gamma_{0}{ }^{2}} \frac{\tilde{J}(y)^{2}}{\tilde{f}_{\infty}(y)}\right) \\
\left(y^{2} \tilde{f}_{\infty}(y) \tilde{H}^{\prime}(y)\right)^{\prime}=2 \tilde{H}(y) \tilde{K}(y)^{2} \\
\tilde{f}_{\infty}(y)\left(y^{2} \tilde{J}^{\prime}(y)\right)^{\prime}=2 \tilde{J}(y) \tilde{K}(y)^{2} \\
\tilde{f}_{\infty}(y)=y^{2}-\frac{1}{y}
\end{gathered}
$$

Let us end this section by noting that the black hole temperature resulting from rescaling (21) and taking the $R \rightarrow \infty$ limit takes the form

$$
\hat{T} \equiv \frac{T}{e H_{0}}=\frac{3 \gamma_{0}}{4 \pi}
$$

\section{Holographic correspondence}

According to the AdS/CFT correspondence [1]-[3], properties of the dual 3 dimensional field theory defined on the boundary can be read from the behavior of the solution of the system in the bulk. In this approach temperature $\hat{T}$ in (531) corresponds to the temperature of the $d=3$ system. 
Let us first consider the vacuum expectation value in the $d=3$ field theory for the scalar operator $\mathcal{O}_{H}$, dual to the field $H$ defined on the bulk. It follows from the identification $\left\langle O_{H}\right\rangle \sim H_{1}$ with $H_{1}$ defined in eq.(27).

The asymptotic behavior of the $A_{0}$ component of the gauge field allows one to identify the chemical potential $\mu$ and the charge density $\rho$ of the 3 dimensional system. Indeed, given the ansatz for the $A_{0}$ component of the gauge field, eq.(12), the $U(1)$ electric field as defined in (34) reads

$$
E_{i}=\mathcal{F}_{i 0}^{U(1)}=\frac{H^{a}(x)}{H_{0}} F_{i 0}^{a}=e H_{0} H(x) \partial_{i} J(x)
$$

(the $\vec{H} \cdot \vec{A}_{0} \wedge \vec{A}_{i}$ contribution coming from the field strength commutator vanishes because $\vec{H} \| \vec{A}_{0}$ ).

Since $\lim _{x \rightarrow \infty} H(x)=1$, the electric field at infinity is given by

$$
\lim _{x \rightarrow \infty} E_{i}=e H_{0} \lim _{x \rightarrow \infty} \partial_{i} J(x)
$$

We have now to expand $J(x)$ for large $x$ and look for the $1 / x$ term which is the one leading to a nontrivial electric flux (i.e., leading to the dyon charge). The corresponding coefficient defines the charge density:

$$
\rho=-\tilde{J}_{1}
$$

Here and in what follow we use the coefficients of the asymptotic expansions defined in $\mathbb{R}^{2} \times S^{1}$. Following [18] we shall define a normal component $\rho_{n}$ by analyzing the expansion for $\rho$ at the horizon using eq.(26),

$$
\rho_{n}=\tilde{j}_{h}
$$

and a superconducting charge density in the form

$$
\rho_{s}=r h o-\rho_{n}
$$

The constant value $\tilde{J}_{0}$ that $A_{\tau}$ takes at the boundary at infinity is related to the chemical potential through the formula

$$
\mu=e H_{0} \tilde{J}_{0}
$$




\section{$5 \quad$ Numerical Analysis}

We shall restrict the numerical calculations to the case in which the boundary is $S^{1} \times \mathbb{R}^{2}$. We shall solve system (50)-(52) using the relaxation method [20] which determines the solution starting from an initial guess and improving it iteratively. The natural initial guess is the Prasad-Sommerfield solution [21] in flat space. Following this procedure, we have found regular solutions for different values of $\gamma_{0}$ i.e. of the temperature $\hat{T}$.

Figures 1 and 2 show a representative dyon solution that exists starting at the horizon. One can see that the scalar rapidly attains its symmetry breaking constant value while the magnetic and electric fields (associated to $K$ and $J$ respectively) concentrate in a spherical shell starting at the horizon. Figure 3 shows a remarkable property of the coupled system of non linear differential equations, namely the existence of several solutions satisfying the appropriate boundary conditions, each one corresponding to a different value of charge density $\rho$. This phenomenon was already encountered in [8] for the case of a $U(1)$ gauge field coupled to a complex scalar in an $\mathrm{AdS}_{4}$-Schwarzschild background. As it also happens in the $U(1)$ case, we have found that for increasing values of $\rho$ the solutions are distinguished by an increasing number of nodes $n$. Now, since evaluation of the free energy shows that it increases with the number of nodes (see below), we conclude that $n>1$ solutions are thermodynamically unfavorable and therefore we shall concentrate hecenforth on the zero node solution.
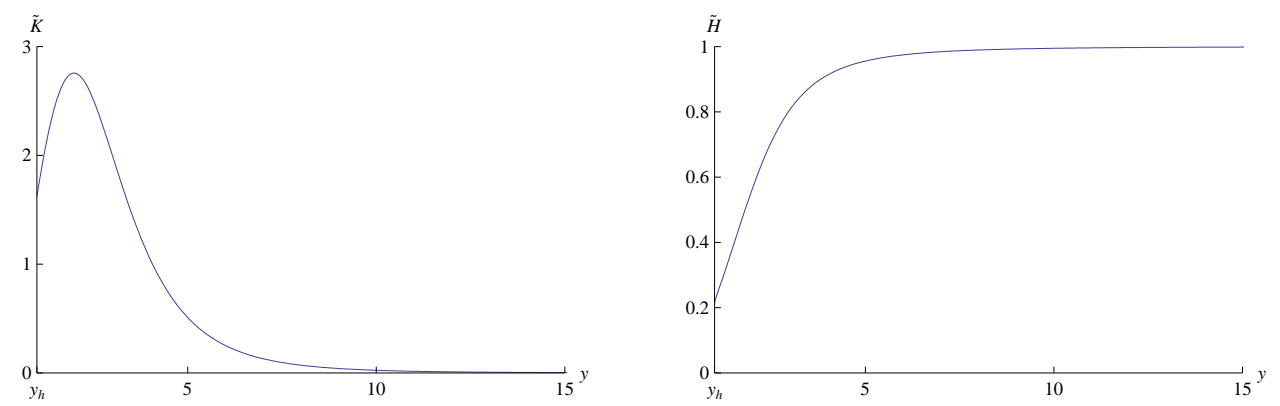

Figure 1: The solution for $\tilde{K}$ and $\tilde{H}$ as a function of $y$ or $\tilde{J}_{0}=6$ and $\gamma_{0}=0.2$ $\left(\hat{T}=3 \gamma_{0} /(4 \pi)\right)$. The solution exists starting at the horizon $y=y_{h}=1$. 


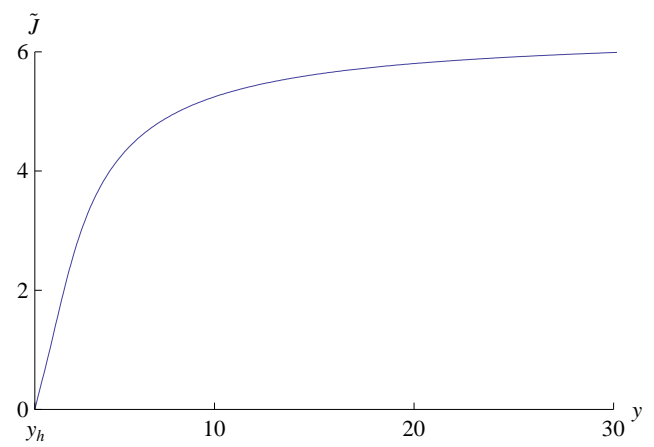

Figure 2: The solution for $\tilde{J}$ as a function of $y$ for $\tilde{J}_{0}=6$ and $\gamma_{0}=0.2$ $\left(\hat{T}=3 \gamma_{0} /(4 \pi)\right)$.

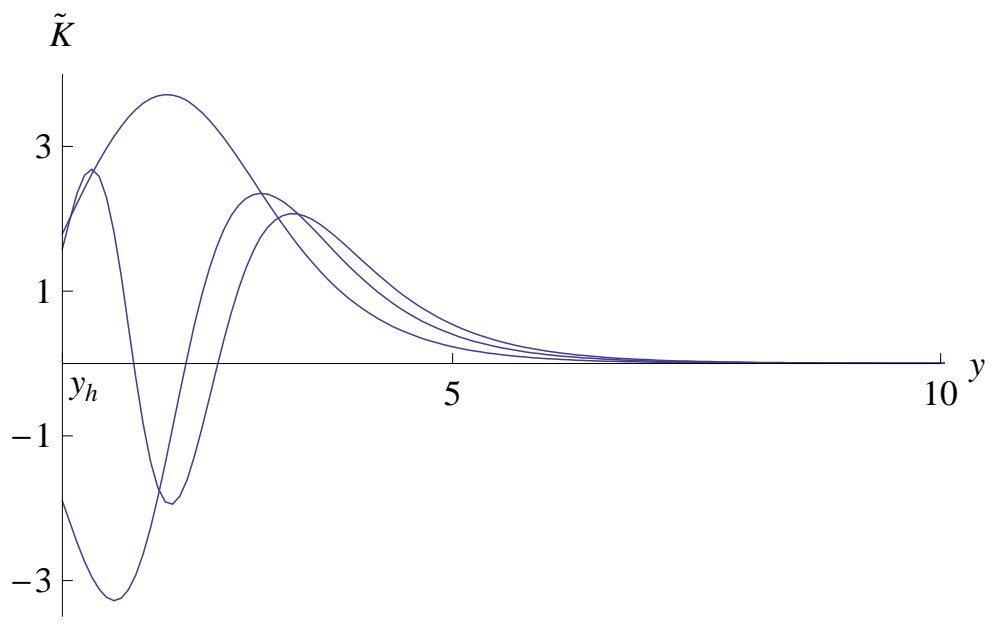

Figure 3: Different solutions for $\tilde{K}$ corresponding to different values of the charge density $\rho$. For increasing values of $\rho$ the solutions are distinguished by the increasing number of nodes.

The behavior of the condensate is shown in figure 4 where the asymptotic coefficient $H_{1} \sim\left\langle O_{H}\right\rangle$ in the asymptotic expansion (27) of the Higgs field is plotted as a function of the temperature (normalized in units of $e H_{0}$ ). In figures 5 and 6 we plot the coefficient $\tilde{K}_{1}$ in the asymptotic expansion of function $\tilde{K}$, while figure 7 shows the fraction $\rho_{s} / \rho$, as defined in eqs.(156)-(158) , related to the behavior of the electric field at the horizon and at infinity. From these figures we conclude that a finite temperature continuous symmetry breaking transition takes place so that the system condenses below a critical 
temperature $\hat{T}_{c}$. By fitting the curves we see that near $T_{c}$ one has a typical second order phase transition with power behavior of the form

$$
\begin{aligned}
\tilde{H}_{1} & \propto\left(\hat{T}_{c}-\hat{T}\right) \\
\tilde{K}_{1} & \propto\left(\hat{T}_{c}-\hat{T}\right)^{1 / 2} \quad \text { as } \hat{T} \rightarrow \hat{T}_{c} \\
\frac{\rho_{s}}{\rho} & \propto\left(\hat{T}_{c}-\hat{T}\right)
\end{aligned}
$$

One can see in figure 4 that at low temperature $\hat{H}_{1}$ appears to diverge. However, one should take into account that when the condensate becomes very large, the backreaction on the metric can no longer be neglected as we did by choosing a fixed Schwarzschild-AdS background metric.

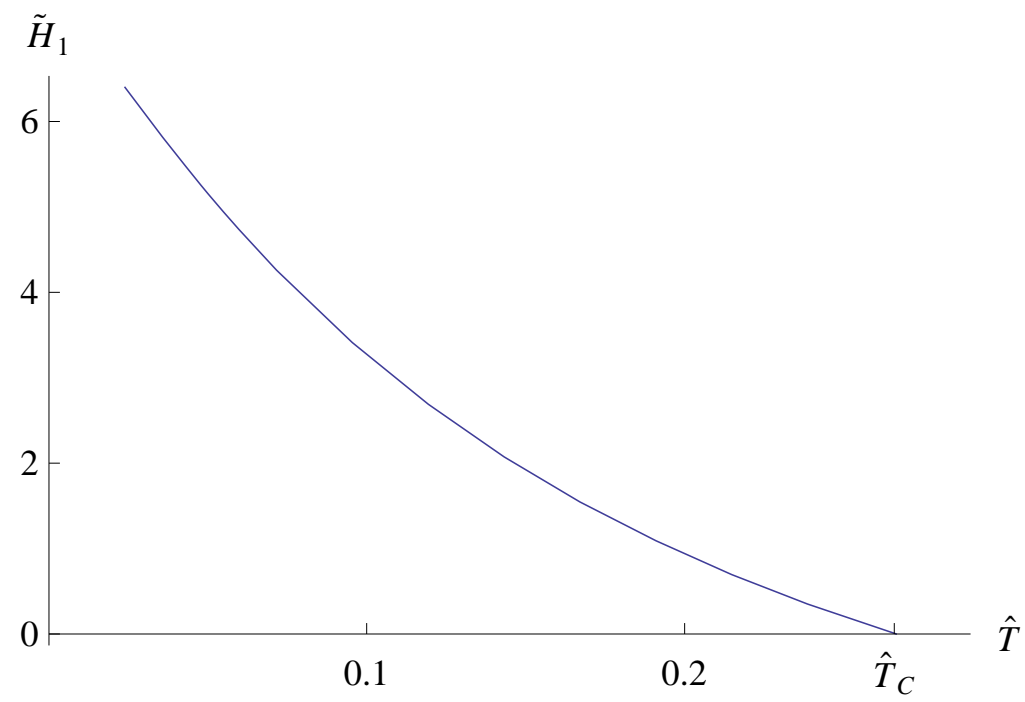

Figure 4: The $\tilde{H}_{1}$ coefficient in the Higgs field asymptotic expansion as a function of the temperature. We have taken $\tilde{J}_{0}=6$ and for this value the critical temperature is $\hat{T}_{c}=0.26649 \ldots$. 


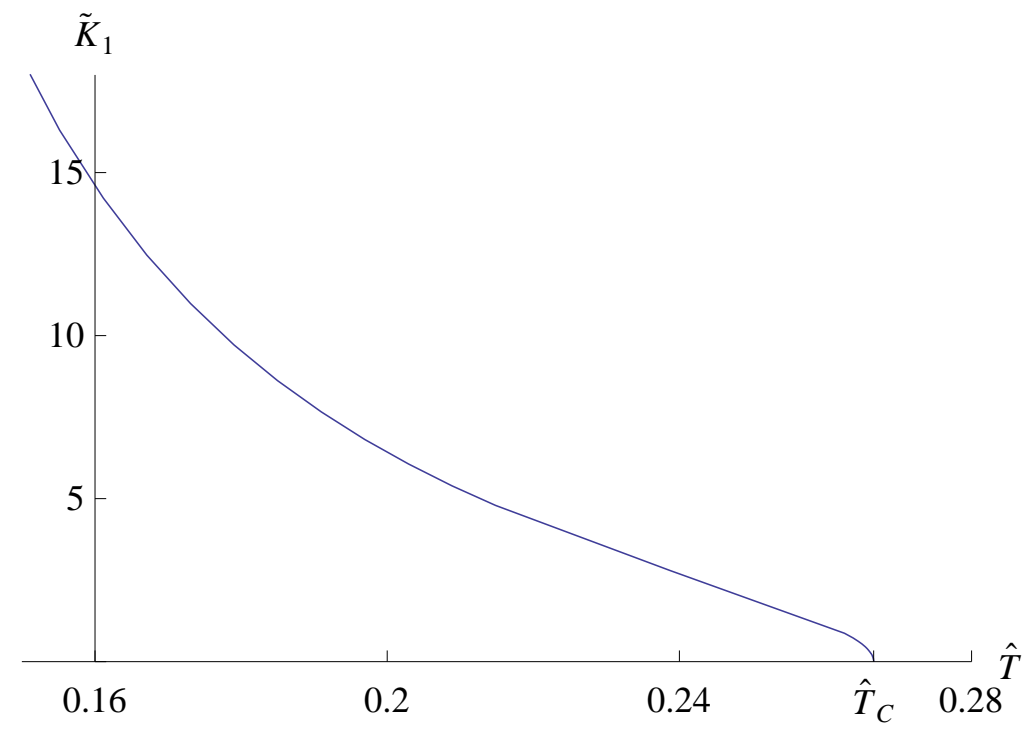

Figure 5: The $\tilde{K}_{1}$ coefficient in the in the gauge field asymptotic expansion as a function of the temperature for $\tilde{J}_{0}=6$.

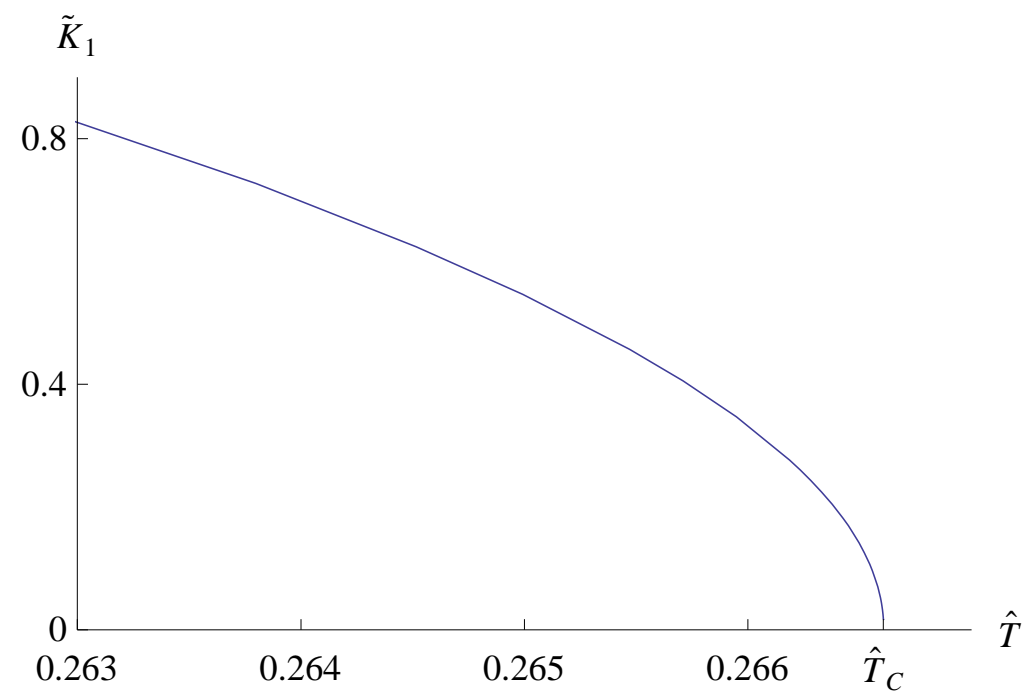

Figure 6: A zoomed view of $\tilde{K}_{1}$ near the critical point. One can see that its behavior near $T=T_{c}$ corresponds to $\sqrt{\hat{T}_{c}-\hat{T}}$. 


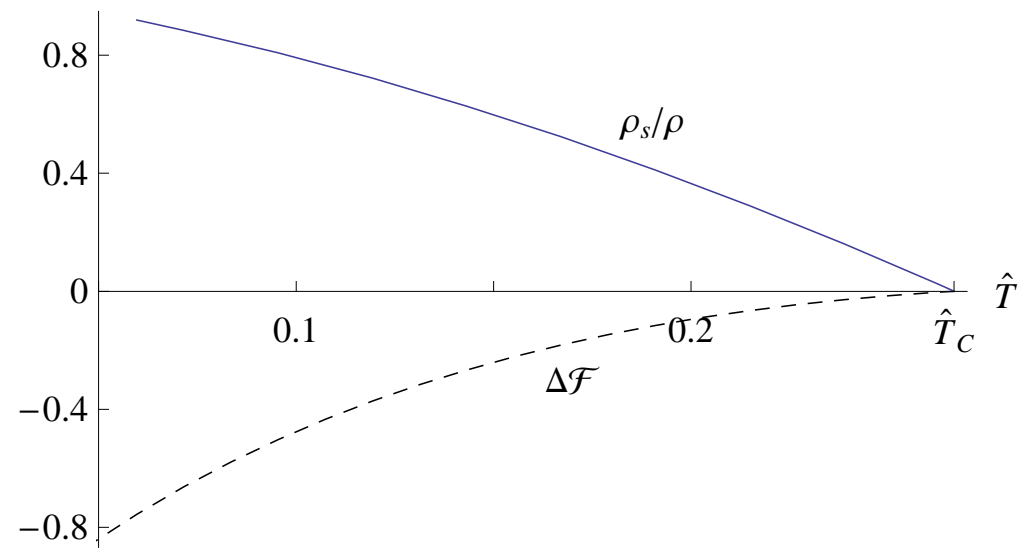

Figure 7: The solid line represents the fraction $\rho_{s} / \rho$ of the superconducting and the total charge densities. The dashed line the free energy difference between the condensed and the uncondensed phases. At $\hat{T}=\hat{T}_{c}, \rho_{s} / \rho$ vanishes linearly.

The free energy $\mathcal{F}$ is given by its on-shell action,

$$
\mathcal{F}=\left.T S_{E}\right|_{\text {on } \text { shell }}
$$

Using the Euclidean action as given in eq.(42) we have numerically computed $\mathcal{F}$ for the dyon solution and compared it with the free energy in the uncondensed phase which corresponds to the solution $\tilde{H}(y)=1, \tilde{K}(y)=$ $0, \tilde{J}(y)=\tilde{J}_{0}(1-1 / y)$. We plot in figure 7 the free energy density difference $\Delta \mathcal{F}$, normalized in units of $e^{2} H_{0}^{4} L / 2$, which can be seen to be continuous at $\hat{T}=\hat{T}_{c}$.

\section{Summary and discussion}

We have applied the AdS/CFT correspondence to describe the strong coupling regime of a $2+1$ quantum field theory which undergoes a phase transition exhibiting the condensation of a composite charge operator below a critical temperature $T_{c}$. The dual gravity theory consists of an $S U(2)$ YangMills-Higgs model in a Schwarzschild-AdS 4 black hole with temperature $T$.

Breaking the gauge symmetry down to $U(1)$ we started by constructing a spherically symmetric soliton solution in the bulk, having both magnetic and electric charge. By choosing appropriate conditions at the horizon and 
at infinity, we found a family of dyon solutions leading to the existence of a condensate, below a critical temperature, in the dual field theory on the boundary which, after an appropriate scaling, becomes $\mathbb{R}^{2} \times S^{1}$.

By calculating the free energy $\mathcal{F}$ we have seen that the condensed phase is thermodynamically favored and we were able to determine which one of the dyon family solutions had the lowest $\mathcal{F}$-value. We have also calculated the power behavior of the condensate, typical of a second order phase transition.

There are many issues that we have not discussed in our work and that deserve a thorough study. To begin with, one should consider the applicability of our results to describe the behavior of an actual condensed matter system. Also, since we have found on the gravity side classical solutions with quantized magnetic flux, we expect that the issue of confinement through the appearence of chromoelectric flux tubes in the dual strongly coupled QFT could take place. In this respect, it should be of interest to consider monopole solutions in Schwarzschild- $\mathrm{AdS}_{5}$ black hole backgrounds so that the dual theory is defined in three spatial dimensions. We hope to discuss theses issues in a future publication.

Acknowledgments We would like to thank Jorge Russo for discussions and helpful suggestions and Tameem Albash for a clarifying comment. A.R.L. is grateful with Borut Bajc for helpful discussions. This work was partially

supported by PIP6160-CONICET, BID 1728OC/AR PICT20204-ANPCYT grants and by CIC and UNLP, Argentina.

\section{References}

[1] J. M. Maldacena, "The large N limit of superconformal field theories and supergravity," Adv. Theor. Math. Phys. 2 (1998) 231 [Int. J. Theor. Phys. 38 (1999) 1113] [arXiv:hep-th/9711200].

[2] S. S. Gubser, I. R. Klebanov and A. M. Polyakov, "Gauge theory correlators from non-critical string theory," Phys. Lett. B 428 (1998) 105 arXiv:hep-th/9802109].

[3] E. Witten, Adv. Theor. Math. Phys. 2 (1998) 253 arXiv:hep-th/9802150]. 
[4] S. S. Gubser, "Phase transitions near black hole horizons," Class. Quant. Grav. 22 (2005) 5121 arXiv:hep-th/0505189].

[5] S. S. Gubser, "Breaking an Abelian gauge symmetry near a black hole horizon," Phys. Rev. D 78 (2008) 065034 [arXiv:0801.2977 [hep-th]].

[6] S. A. Hartnoll, C. P. Herzog and G. T. Horowitz, "Building a Holographic Superconductor," Phys. Rev. Lett. 101 (2008) 031601 arXiv:0803.3295 [hep-th]].

[7] S. S. Gubser, "Colorful horizons with charge in anti-de Sitter space," Phys. Rev. Lett. 101 (2008) 191601 [arXiv:0803.3483 [hep-th]].

[8] T. Albash and C. V. Johnson, "Phases of Holographic Superconductors in an External Magnetic Field," arXiv:0906.0519 [hep-th]; "Vortex and Droplet Engineering in Holographic Superconductors," arXiv:0906.1795 [hep-th].

[9] M. Montull, A. Pomarol and P. J. Silva, "The Holographic Superconductor Vortex," Phys. Rev. Lett. 103 (2009) 091601 arXiv:0906.2396 [hep-th]].

[10] S. S. Gubser and S. S. Pufu, JHEP 0811 (2008) 033 arXiv:0805.2960 [hep-th]].

[11] R. Manvelyan, E. Radu and D. H. Tchrakian, Phys. Lett. B 677 (2009) 79 arXiv:0812.3531 [hep-th]]; S. Franco, A. Garcia-Garcia and D. Rodriguez-Gomez, arXiv:0906.1214 and 0911.1354 [hep-th]; K. Peeters, J. Powell and M. Zamaklar, JHEP 0909 (2009) 101 arXiv:0907.1508 [hep-th]]; K. Goldstein, S. Kachru, S. Prakash and S. P. Trivedi, arXiv:0911.3586 [hep-th]; F. Aprile and J. G. Russo, arXiv:0912.0480 [hep-th].

[12] A. R. Lugo and F. A, Schaposnik, Phys. Lett. B 467 (1999)43.

[13] A. R. Lugo, E. F. Moreno and F. A, Schaposnik, Phys. Lett. B 473 (2000)35.

[14] G.W. Gibbons, Lectures given at 12th Lisbon Autumn School on Physics, Lisbon, Portugal, Oct 1-5, 1990. Published in Lisbon Autumn School 1990:110-133. 
[15] A. M. Polyakov, "Particle spectrum in quantum field theory," JETP Lett. 20 (1974) 194 [Pisma Zh. Eksp. Teor. Fiz. 20 (1974) 430].

[16] S. W. Hawking and D. N. Page, "Thermodynamics Of Black Holes In Anti-De Sitter Space," Commun. Math. Phys. 87, 577 (1983).

[17] E. Witten, "Anti-de Sitter space, thermal phase transition, and confinement in gauge theories," Adv. Theor. Math. Phys. 2 (1998) 505

[18] S. S. Gubser and S. S. Pufu, "The gravity dual of a p-wave superconductor," JHEP 0811 (2008) 033 [arXiv:0805.2960 [hep-th]].

[19] B. Julia and A. Zee, "Poles With Both Magnetic And Electric Charges In Nonabelian Gauge Theory," Phys. Rev. D 11 (1975) 2227.

[20] W. H. Press, S. A. Teukolsky, W. V. Vetterlink, Numerical Recipes: The art of Scientific Computing, Cambridge University Press, Cambridge UK, 1992.

[21] M. K. Prasad and C. M. Sommerfield, Phys. Rev. Lett. 35 (1975) 760. 